\title{
Single Photon Emission Tomography (SPECT) and 3D Images Evaluation in Nuclear Medicine
}

\author{
Maria Lyra \\ University of Athens
}

Greece

\section{Introduction}

The target of Nuclear Medicine is to provide information on the distribution of a chosen molecule in space and/or also in time, inside the human body. An image of the distribution, of a molecule of biochemical or physiological importance, within the body in a specific organ, provides information on the functioning of an organ that is valuable for medical diagnosis and for monitoring the response to the treatment of a disease.

The techniques used in Nuclear Medicine involve labelling of a specific molecule with a radioactive atom; a quantity of which is administered to the patient. The labelled molecules follow their specific biochemical pathways inside the body. The atoms used are radioactive and undergo random decay, emitting gamma ray photons. Photons are detected outside the body by the detector $-\mathrm{NaJ}(\mathrm{Tl})$ crystal- of the gamma camera.

Imaging by two-dimensional (2D) planar scintigrams has been routinely used since many years. The functional information obtained by Single Photon Emission Tomography (SPECT) is complementary to the planar images, obtained by projections of the organ under investigation. SPECT is an important tool for evaluating the functional status of the human body, emerging information by reconstructing data in slices of the total organ.

Image quality and quantitative accuracy in SPECT can be degraded by some parameters as the effects of photon attenuation and finite spatial resolution of the tomographic gamma camera system.

Volume data in nuclear medicine, by rendering and shading, give the 3 dimensional (3D) description of an organ and carry information of an organ's surface in angles around it. They are obtained by a sequence of $2 \mathrm{D}$ slices reconstructed from projections acquired around a 3D volume organ. Volume visualization obtains volumetric signs useful in diagnosis, in a more familiar and realistic way. Filtering, thresholding and gradient are necessary tools in the production of diagnostic 3D images.

The required input data for creation of $3 \mathrm{D}$ surface images is a dataset containing slice images which have been previously reconstructed from the SPECT acquisition data. These slices may be in one of several planes: transaxial, sagittal, coronal or oblique, and the orientation of the input data determine the orientation of the final 3D surface dataset. The $3 \mathrm{D}$ reconstruction of external surface of kidneys, lungs, liver, thyroid and heart are described here, focused on the details and precision of the final surfaces through grading.

Source: Image Processing, Book edited by: Yung-Sheng Chen,

ISBN 978-953-307-026-1, pp. 572, December 2009, INTECH, Croatia, downloaded from SCIYO.COM 


\section{Volume data in nuclear medicine}

Organ volumes evaluation using planar imaging technique is a procedure often performed in nuclear medicine but faces difficulties due to structures containing radioactivity, which overlie or underlie the organ of interest. SPECT overcomes these difficulties since structures in the interior of the examined organ are separated in the reconstructed images (Rosenthal et al, 1995).

The presentation of functional volumes and activity concentrations in SPECT requires that a linear and stationary relationship exists, between the apparent activity distribution in the reconstructed image and the true distribution in the organ. However, because of the limited spatial resolution of the gamma camera, and physical processes such as photon scattering and photon attenuation, this relationship is neither linear nor stationary (Gourion \& Noll, 2002).

Absolute quantification of the 3D distribution of a radionuclide within a patient has been one of the greatest challenges of Nuclear Medicine. This is difficult due to the fact that nuclear medicine images are degraded by several factors (anatomic, physical, technical factors), which limit the quantitative ability of this modality (Tsui et al, 1994).

SPECT is used in image analysis studies to extract information about areas and volumes or amounts of radioactivity in specific regions of interest. The information that derived from these studies is then applied to aid in clinical diagnoses or even to estimate radiation dosimetry or determination of volume or mass (De Sadeleer et al, 1996; Erdi et al, 1995; Pant et al, 2003;). Many methods for precise and accurate measurements of volume and the amount of radioactivity in a specific region of interest are used (Boudraa \& Zaidi, 2006; Hansen, 2002a).

The set of SPECT images (slices) is used for the 3D volumetric representation of internal organs of the human body. Image based volume rendering techniques have demonstrated the improvement of rendering quality when $3 \mathrm{D}$ organ presentation is based on digital images as SPECT slices and some shape and functional information (Sainz et al 2003). 3D volume representation may reach photorealistic quality taking into consideration the factors that degrade slices data and affect quantitative accuracy (Zaidi, 1996b).

\subsection{Data acquisition}

3D images, in nuclear medicine techniques, are created by acquisition data sets in angular planes. The data are acquired by SPECT gamma camera in an arc of 180 or 360 degrees depending on the size and the position of the studying organ- and multiple slices that can be treated as volume data are produced from the angular projections.

It is often considered that adequate compensation for the effects of physical factors, as non uniform attenuation or distance-dependent spatial resolution, requires data acquired over $2 \Pi$. However, in many cases the data measured over $2 \pi$ contain redundant information. Sometimes, $2 \Pi$ acquisition affects the quality due to effect of partially compensating attenuation of the surrounding the organ tissues. Noo \& Wagner, 2001 have shown that data acquired only over $\Pi$ can be used to correct for the effect of uniform attenuation in SPECT and Pan et al, 2002 showed that with both non-uniform attenuation and distance- dependent spatial resolution the scanning angle can be reduced from $2 \Pi$ to $\Pi$.

Thyroid gland is a front superficial organ and best data are collected by a 180 degrees arc acquisition, in a clockwise direction. In the case of kidneys - a posterior organ- data are acquired in a counter clock direction, over п. Similarly, because of heart position in the left 
part of the chest, the data for reconstruction are acquired in a clockwise direction, from $-45^{0}$ to $135^{\circ}$, that is, the data are acquired over $\Pi$.

Pan et al, 2001 are referred to mathematical rationales that explain that $2 \Pi$ measurements contain redundant information, as, in the absence of noise and other inconsistencies, the measurements of conjugate views are mathematically identical, i.e.

$$
\mathrm{p}(\xi, \varphi)=\mathrm{p}(-\xi, \varphi+\Pi)
$$

This is an example of the measurement of the 2D Radon transform $p(\xi, \varphi)$ of a real function over $2 \Pi$, where $\xi$ is the detector bin index and $\varphi$ is the angle acquiring data.

In any cases of human organs, as lungs and liver, that their anatomic location includes front and posterior regions, the $2 \Pi$ geometry during data acquisition and reconstruction is necessary.

\subsection{Attenuation}

It is assumed that the count rate measured at a point on a planar image is equal to the sum of the count rates emerging from all the volume elements along the ray line. In reality a significant number of gamma rays will be scattered or absorbed before reaching the detector. This results in a reduction in the observed count rate at the detector:

$$
\mathrm{N}=\mathrm{N}_{1} \cdot \mathrm{e}^{-\mu \mathrm{d}}
$$

where $\mathrm{N}$ is the measured count rate when $\mathrm{N}_{1}$ is the count rate which would be measured if there were no attenuation, $\mathrm{e}$ is the base of the natural logarithms, $\mathrm{d}$ is the thickness of attenuating material through which the gamma rays must pass, and $\mu$ (attenuation coefficient) is a constant which depends on the energy of the gamma rays and the type of attenuating material. For $140 \mathrm{KeV}$ gamma rays of Tc99m -the radioisotope more used in Nuclear Medicine- the attenuation coefficient is $\mu=0.15 / \mathrm{cm}$, for human tissue; because of scatter a value of $0.12 / \mathrm{cm}$. is usually used to correct for attenuation( Rosenthal et al, 1995).

A procedure is used for correcting of the errors in reconstruction introduced by attenuation. The attenuation correction procedure is a first order method. Because of the inaccuracy of the correction procedure it is necessary to use a value less than the theoretically correct value; $0.12 / \mathrm{cm}$ is usually best for technetium gamma rays in man (Zaidi, 1996; Zaidi \& Hasegawa, 2003).

If attenuation correction is used the system needs to know the extent of the attenuating material that is the edge of the patient. The patient outline can be calculated by the system. A threshold level is calculated, many times, as a percentage of the maximum pixel intensity for the whole planar dataset.

\subsection{Volume rendering}

Volume visualization in nuclear medicine is a method of extracting meaningful information from volumetric data using, manipulating and rendering a sequence of 2D slices. Volume data are 3D entities that may have information inside them or at there surface and edges at angular views that can be obtained by 3D surface rendering images in different angular views. During rendering time (Sainz et al 2008), given a new view point, the step is to determine which of the reference (acquired) views contribute to the new one and then if they overlap, combine them to produce the final volume (fig.1). 


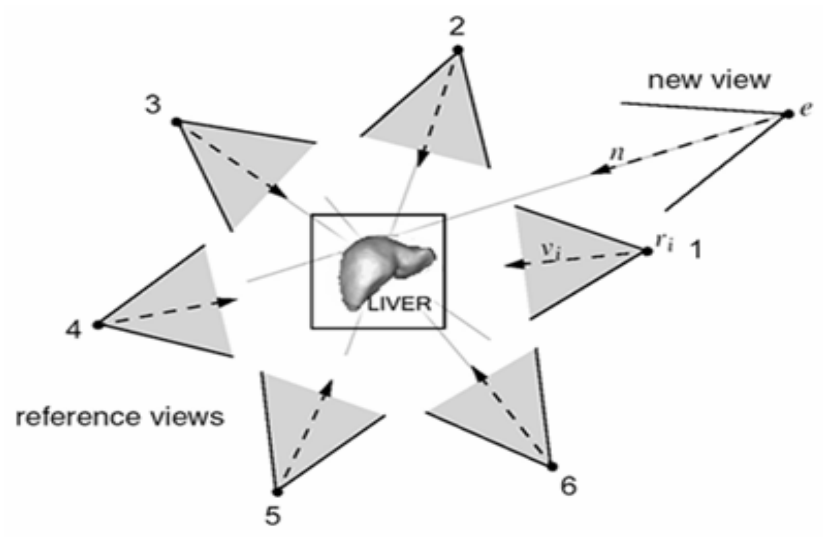

Fig. 1. Distribution of angular acquired data. Reference Views will contribute to the interpolated new view. (from Sainz et al 2008)

3D images are reconstructed by surface rendering techniques. Organ surface volumes in nuclear medicine can be made from the scientific data by outlining (threshold use) structures in the serial slices and after stacking them, surface reconstruction is done. The result of tomographic reconstructions is a set of contiguous or superposed slices in which the basic element is a voxel, since it represents volume, with the third dimension equal to one pixel. Voxels are reorganized and displayed in 3D way by labeling each voxel with information concerning the distance and the angle of its surface. Surface images are created and interpolated for any angle resulting in rotating surface images.

By gathering sufficient volume data, analysis and quantification of 3D images give valuable information in medical diagnosis. Angular measurements on body structures are often crucial for analysis of the data for diagnosis. Shaded surface images provide unique views of the three dimensional radiopharmaceutical distributions within the human body (Shin et al, 2009).

When viewed together with two-dimensional data, 3D surface images give excellent information about the location, orientation and extent of regions of isotope uptake. 3D datasets are very strong visual representations of tomographic data, and are mainly used qualitatively to help orientate the user with the tomographic slice data. This reinforces the three dimensional nature of nuclear data and strengthens the user's awareness of what the data represents and he can glean information on perfusion details for liver or cardiac studies and function information of thyroid, lungs or kidneys, from the 3D surface images.

When 3D surface images are generated, data are processed from each slice in order. This set of slice images contains information in the form of count values in volume elements, voxels, which represents the distribution of the radiopharmaceutical within the volume being imaged. The 3D surface images which are produced provide views of a specific three dimensional surface of this volume. This surface is defined by a count threshold which is applied to each of the voxels; all voxels which have count values greater than the threshold define a specific volume. The surface images display the surfaces of this volume. There are 3 possible ways in which 3D datasets can be generated: a) At a fixed count threshold, the view angle of the surface is varied to create the impression of a rotating surface, b) At a fixed viewing angle, the count threshold is varied to create a dataset which sequentially removes 
layers of counts from the object rather like peeling the layers from the examined organ, c) Using a fixed view angle and threshold, the distance from which the surface is viewed is varied. This enables the user to take slices through the surface image.

\subsection{Thresholding}

Many methods have been developed for image edge detection and most of these techniques work well in images with uniform regions, but less well in regions with greater non uniformity. Medical images are usually characterized by faded features utilizing a narrow distribution of grey-levels. Nuclear medicine images often suffer from low contrast that is further degraded by the noise introduced in the process of imaging (Razifar et al 2005). It is important to use the local contrast information as well as the overall distribution of greylevels for obtaining meaningful regions. A large number of algorithms with many approaches have been investigated. These approaches include histogram based methods, edge and boundary detection based methods, region growing based methods, and linear feature based methods (Mortelmans et al, 1986; Russ 2006; Murli et al, 2008).

The accurate reconstruction of organ volumes from SPECT images suffers from image segmentation. Image segmentation defines the borders of the organ and allows volume measurements by counting the voxels inside (Zingerman et al 2009). The threshold has to be adapted for each case as it is dependent on the size of the organ and the contrast of the reconstructed SPECT slices.

Some threshold algorithms have been applied to minimize the difference between true and reconstructed volumes. Grey level histograms analysis has been proven a suitable technique for thresholding. Segmentation is performed with algorithms that separate object and background pixels by comparing their intensity (counts) with the mean intensity of the region. A region of interest is roughly defined by the operator to include the slices of the organ to be reconstructed.

Thresholding is one of the simplest and popular techniques for image segmentation. It can be performed based on global information that is grey level histogram of the entire image or using local information of the image. In thresholding method, to obtain automatically the optimum threshold value, an initial estimate for threshold $\mathrm{T}$ is selected. The image is segmented using T.(Boudraa \& Zaidi, 2006). In this way two regions of pixels will be separated: $R_{I}$ all pixels of the image with grey values $>T$ and $R_{B}$ the pixels of the region considered as background with values $<T$. By the average grey level values $M_{I}$ and $M_{B}$ for the regions $R_{I}$ and $R_{B}$ a new threshold value is computed:

$$
\mathrm{T}_{\mathrm{N}}=\left(\mathrm{M}_{\mathrm{I}}+\mathrm{M}_{\mathrm{B}}\right) / 2
$$

The main drawback of histogram-based region segmentation is that histogram provides no spatial information. Region thresholding approaches exploit the fact that pixels close to each other have similar grey values. The main assumption of this approach is that regions are nearly constant in image intensity and it is referred as a robust technique for volumetric quantification and localization of abnormal regions.

However, combining intensity and gradient data can improve histogram (Csetverikov, 2007). Better separation of objects and background pixels close to edges, give high gradients; pixels of object and background, though, have low gradients. Then, in order the result be improved, histogram high-gradient pixels are discarded. (fig.2.). 
The gradient shading is used to add shading to the 3D reconstructed image Gradient shading enhances the display of surface detail.
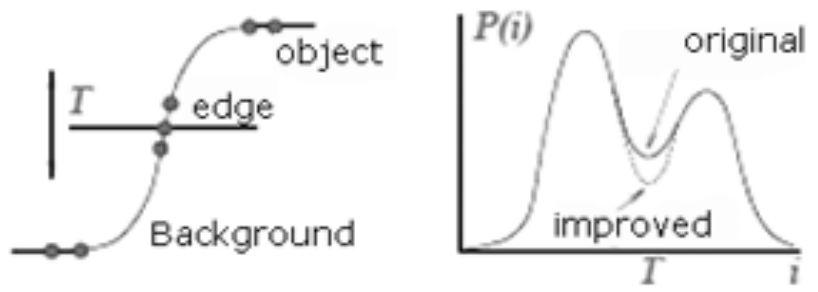

Fig. 2. Discarding high gradient pixels close to edges improves the reconstructed image.

(from Csetverikov, 2007)

\section{The reconstruction process}

The basic reconstruction is created by the planar images, collected by the gamma camera and contain the number of gamma rays detected at each angle position on the face of the collimator. The image which is to be generated is composed of the numbers of gamma rays emitted from each of the small pixels. The data from which this are to be calculated is the sums of the counts in various rows of the pixels drawn at the angles at which images were recorded at acquisition time.

If, for example, 64 planar images in $64 \times 64$ matrices are acquired $360^{\circ}$ around the patient, each transaxial slice can be reconstructed from the information taken from a single row of elements (pixels) in each planar image. This information is used to calculate the values of 4096 pixels in the transaxial slice. For each angle-each planar image- there are 64 measured values, each measured value being the sum of the numbers which should appear in the reconstructed slice. The reconstruction problem is to calculate a set of numbers for the transaxial matrix the sums of which, in every direction at which a measurement was made, are equal to the measured values at that angle. In the current example there are 4096 equations (64 sums multiplied by 64 angles) containing a total of 4096 unknowns, the numbers in the pixels.

\subsection{Filtering}

A number of methods have been used to perform reconstructions of this type.

The method of Filtered -Back-Projection (FBP) is composed of two stages; the first is filtering of the planar image data, and the second is to back-project the filtered data to form transaxial images. The back projection is based on the inverse transformation of the geometrical model of the SPECT data acquisition. The algorithm works well for infinite number of projections, but when a limited number of projections are produced the back projection method causes "star pattern" around the reconstructed object. The star-shaped pattern and the noise are reduced by filtering the projections with a FBP algorithm (fig.3).

When the collected data are back projected, the rows with the largest numbers overlap at the position of the source, but not elsewhere. The result is that the back-projected numbers add up over the position of the source into very much larger numbers than those in other areas of the matrix. 


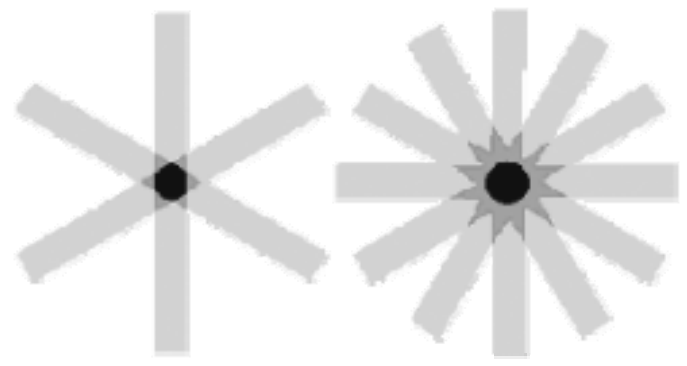

Fig. 3. Back projections of a point source created by finite numbers of projection angles; in this case "star pattern" occurs.

The difficulty with simple back-projection is that areas of the reconstructed slice which should be zero, as no radioactivity was there, are not zero. In addition there is always an increase in the numbers near the centre of the image. In order to overcome these problems, the original data are necessary to be modified by "filtering" before back-projecting.

\subsubsection{Ramp filter}

The modification procedure is done by filtering. This additional filtering can be performed by pre-filtering the planar data, by filtering the final reconstructed slices, or by combining a suitable filter with the back-projection filter. Because of these, it is convenient to perform the filtering on the Fourier transform of the data, rather than on the data itself.

The filter which is used is a frequency filter. It causes some spatial frequencies to be enhanced and others to be reduced. Correction for the central build-up in back-projection requires that low spatial frequencies be attenuated and high spatial frequencies be amplified.

The filter which exactly corrects for the back-projection defects is the Ramp filter (fig.4). This filter enhances high frequencies by multiplying all frequencies by a term proportional to the frequency. This "Ramp filter" compensates for the distortion introduced by back-projection but takes no account of the nature of the information being back-projected. The ramp filter may be applied in two ways; one takes account of several mathematical considerations, producing quantitative results and the other produces results with improved image contrast

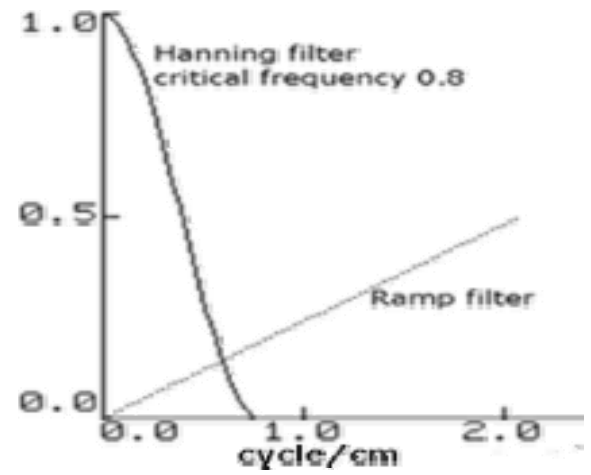

Fig. 4. High frequency information is greatly magnified by the Ramp filter. Hanning filter drops sharply to zero at a frequency $(0.8 \mathrm{cycle} / \mathrm{cm}$, in this figure) close to the limiting resolution of the gamma camera. 


\subsubsection{Filtering approaches}

Radioactive decay is a random process, so all gamma camera data is limited by counting statistics. The statistical variations which always exist between one pixel and the next tend to disguise the true count rate being measured. This statistical noise can vary rapidly from one pixel to another according to Poisson statistics, and so contains high spatial frequencies. The information contained in these high frequencies is not real information about the patient, but contains "noise". This statistical noise is not wanted, but is always present in gamma camera images. Although it may be necessary to tolerate statistical noise in most images, there is no desire to increase the amount of noise. If tomographic data is filtered using the Ramp filter, all high frequency information is greatly magnified, and so therefore is the statistical noise (fig.4). For this reason the filters used in tomography are usually designed to drop sharply to zero at a frequency which corresponds to the limiting resolution of the gamma camera. Two general classes of such filters are provided, in addition to the ramp filter. The user may choose the limiting frequency at which the filter goes to zero as by Hanning filter (fig.4) or the relative height of the filter in the mid-frequencies when it starts to approach zero by Butterworth filter. Kao \& Pan, 2000, have described other non-iterative methods that suppress image noise and artifacts in 3D SPECT.

Iterative reconstruction methods are also used to obtain images with good signal-to-noise ratio in nuclear medicine image reconstruction. Maximum Likelihood Expectation Maximization (ML-EM) method reduces artifacts but requires more computation time. Another iterative filtering method the Ordered Subsets Expectation Maximization (OSEM) is used in 3D SPECT reconstruction and compared to FBP. OSEM and FBP presented similar accuracy in volume measurements of myocardial perfusion (Daou et al, 2003). However, it is possible to calculate the noise constructed by FBP while this not possible by iterative methods (Hansen, 2002b). ROC analysis of OSEM and FBP techniques in lung cancer, showed that there was no significant difference in the area under the ROC curve (Son et al, 2001).

\section{Surface images process}

The imaged volume data which will be used for surface image generation is obtained from a set of transaxial, sagittal, coronal, or oblique slice images. Prior to the generation of the surface images, processing operations should be performed.

To improve the resolution of acquired data the maximum possible matrix size both for data acquisition and processing is used. The visual quality of the surface images improves as the voxel size decreases. Therefore, a $128 \times 128$ resolution acquisition would be preferred and is used for acquiring data from organs as kidneys or thyroid gland. In practice, however, many times a $64 \times 64$ acquisition is used with excellent results; The use of the $64 \times 64$ matrix offer the advantage of reduced storage requirements and processing times. Data from organs as myocardium or lungs are usually acquired in a $64 \times 64$ matrix size.

Filters used in reconstruction are also very important for the maximum transfer of information in 3D images. The choice of the filter is dependent upon the noise characteristics of the data (Yan and Zeng 2008). Since the images depict a surface, an increased amount of smoothing may be desirable without loosing surface special features, as roughness or smoothness, which may be characteristics of an abnormality of the organ. The appearance of defects at parts of the organ that are not functioning is crucial too. Lungs emboli follow up by imaging, for the perfusion improving or renal pelvis imprint are signs for the diagnosis 
and must be emerged in surface 3D images. A good general purpose filter for this application is the Hanning 0.8 prefilter, with a ramp backprojection filter. For high resolution data such as thyroid studies, a Butterworth filter which passes more of the middle frequencies can be used.

Zoomed reconstruction technique is used to perform a 3D zoom in the transaxial space in cases of small organs as pediatric kidneys or thyroid gland. Any tomographic slice dataset may be magnified in 3 dimensions, following reconstruction. Three dimensional magnification is recommended as it reduces the voxel size, providing improved visual image quality. Since the output images have the same resolution in pixels as the input images, magnification is limited by the possibility of excluding volumes of interest.

A magnification by a factor of 2 in all three directions is common in small organs. For example, for an initial set of 32 transaxial slices of $64 \times 64$ resolution data, as in the case of a myocardium SPECT acquisition in an 1800 arc, the portions of the transaxial images within a certain window are magnified by double. In addition, 31 new magnified transaxial slice images are created in between each of the magnified original images. The resulting dataset will contain 63 transaxial slice images of $64 \times 64$ data.

It is possible to magnify the set of slices which is the result of a previous magnification, to obtain an over-all magnification factor of 4 . In this study, myocardium perfusion transaxial slice data is normally magnified by a factor of 2 prior to 3D surface image generation.

In cases where multiple structures exist in the imaged volume, it may be useful to remove data which does not belong to the structure of interest by selectively remove unwanted structure from the slice images by setting selected voxel values to zero. This operation has applications in lung studies, to separate the right and left lungs or in studies of the liver where the spleen may be useful to be zeroed out. It is, also, sometimes, useful to apply in kidneys 3D surface images creation, in cases that the function of the parenchyma of one kidney is very deteriorated and the maximum intensity of one kidney is lower than $50 \%$ of the other kidney. In this case, two regions of interest are defined for the two kidneys and are processed separately. Then each kidney is reconstructed, determining the threshold as percentage of the its maximum.

\subsection{Distance shading procedure}

3D Surface images are created from tomographic slice data in two steps in which the images are shaded. By distance shading operation, the distance shaded surface images are produced. The detection of the surfaces occurs and this accounts for most of the processing time. The surface which will be defined for display is the surface of a volume which is obtained using a calculated count threshold. This volume contains all voxels, which have count values which are above the count threshold value. The distance surface images are created to appear as if the volume is being viewed from specific viewing planes. In the creation of 3D surface images of all the studied organs, the data of transaxial slices were used; by this selection, the orientation of the organs in the body (head/up- feet/down) is kept.

A transaxial slice image is displayed, after the application of a threshold intensity/count value so that all voxels which have count values which are greater than the count threshold are white and those below are black. The outer edge of the threshold slice is the desired surface boundary. A distance surface is generated from the entire set of slices.

The 3D image which is displayed is the surface of a volume defined by count threshold. Thus, volume contains all voxels which have count values which are above the count 
threshold value. For ease in application, the calculated, according to equation (3) threshold level is specified as a percentage of the maximum count level in the input slice dataset.

It is helpful when determining the appropriate threshold value for a given dataset, to create multiple surface images, from one viewing plane, changing only the threshold value from one image to the next, iteratively.

Typical threshold values are usually of the order of $40 \%-50 \%$ for heart, lungs and liver data. For kidneys and thyroid gland data may give a different value, ranging from $30 \%$ to $50 \%$.

\subsection{Viewing plane}

The distance shaded surface images are created to appear as if the volume is being viewed from specific viewing positions. Each position is more specifically defined as an image which lies in a predefined viewing plane. These viewing planes may be positioned anywhere around the imaged volume, if the vertical axis of the viewing plane is parallel to the axis which is normal to the slice data. As the slice images are of the transaxial type, the viewing planes are positioned to view the data from the same locations as in the original projection/ planar views, at any angular position about the axis (Garcia et al, 2001).

The angular position of the 3D surface images depends on the angle steps (usually 16) selected to reconstruct and view the 3D surface images, as an angle measured clockwise from a line which rises vertically from the centre of the image. Thus, if the input slices are of the transaxial type, 0 degrees corresponds to an anterior view, 90 degrees produces a left lateral view, 180 degrees produces a posterior view, and 270 degrees produces a right lateral view. A start distance value equal to zero places the viewing plane outside the entire organ volume for viewing the surface of the organ.

\subsection{Gradient shading}

Following, gradient shading is used to add shading to the image produced in distance shading procedure. The amount of gradient shading on a set of surface images is varied, without having to re-compute 3D distance shading images, each time. Gradient shading is expressed in percentage and is depending on the surface functionality which in this way expresses.

Gradient shading is used to enhance the display of surface detail. Garcia-Panyela \& Susin, 2002 used surface reconstruction in their dynamic model to provide volume and give functionality keys about the organ. With this type of shading, the surface image brightness is a function of the local slope of the surface. This calculation is carried out on the distance surface images.

The value for the gradient surface image pixel is at a maximum when the local surface element is facing front. As the surface slopes away towards another angle, the gradient image pixel value decreases. In the limit, when the local surface element appears as an edge the gradient image pixel value is zero.

The final 3D surface images produced include the effects of both distance and gradient shading. We have computed 3D gradient shaded images from 3D distance shaded images for all human organs that we are referred in this work. We select the amount of gradient shading which will be added to a distance surface image by a gradient shading factor 10 to 20 depending on the physiology of the organ; that is abnormality of its parenchyma or its perfusion deterioration. The amount of shading to add depends on the black and white or color maps employed. A grey scale map or a color map is used as this is the way that $3 \mathrm{D}$ surface images produce best detailed display useful in medical diagnosis. 


\section{Clinical applications}

The imaging system is a GE starcam AT 4000 equipped with a low energy high resolution collimator (LEHR) for acquisition of scintigraphic studies of all the organs. System collimator, used in all our studies, was this LEHR in order to achieve the most detailed data.

The tomography hardware/software gamma camera facility uses the tomography ring stand, to rotate the camera around the patient during acquisition. A very fast, array processor is used to reconstruct the tomographic images. Furthermore, the whole acquisition- processing system is interfaced with a GE Xeleris 2 for fast computing and displaying planar, SPECT and 3D volume images. Elementary voxel size is determined separately. To establish this measurement, an image of two $1 \mathrm{~mm}$ parallel line sources, $10 \mathrm{~mm}$ apart, was created and the number of pixels between the center of the two line sources was measured. Rotating the line sources 90 degrees would provide the pixel width in the opposite direction. Pixel size was controlled weekly and in a $64 \times 64$ matrix is approximately $6.4 \mathrm{~mm}(+-0.2)$.

The SPECT and 3D reconstructed images of clinical applications of the Heart, Lungs, Liver, Kidneys and Thyroid gland have been exploited and displayed. The application of this technique includes the study of parenchymatous organ (Liver, Kidneys, Thyroid gland) for possible abnormalities or for the potential assessment of organ perfusion (Heart, Lungs) in a three dimensional display. Volume images are rotated on any spatial plane and provide rich detailing of the organ surface and functional completeness. Angular appearance of body structures is often crucial for determining the medical diagnosis.

\subsection{Renal studies- 3D surface images}

Tc-99m DiMercaptoSuccinic Acid (Tc-99mDMSA) renal scan is the method of choice for the detection and follow-up of any possible cortical damage to the kidneys. The test is widely performed in children in order to check for any possible signs of acute pyelonephritis, scars as well as for any suspected dysplasia of the kidneys (Temiz et al 2006). Kidneys' are located in the posterior abdomen in a very small depth depending of the size, weight and age of the patient.

The usual procedure involves intravenous injection of Tc99m-DMSA and acquisition at 4 to 6 hours post-injection, of four planar images: Posterior (POST), Anterior (ANT), Right Posterior Oblique (RPO) and Left Posterior Oblique (LPO). The planar imaging (renal scanning) of the patients is followed by a SPECT study (Groshar et al, 1997; Yang et al, 2001). Tomographic imaging was carried out by data acquired in $15 \mathrm{sec}$ at each of 32 positions over 180 degrees around the posterior view of the patient. The 32 angular projection views - the original data as recorded by the gamma camera- are reconstructed to get SPECT slices transaxial, coronal and sagittal-. An acquisition magnification equal to 2 was used for planar as well as angular projections for the SPECT study. Acquisition matrix size 128x128 was used in all cases and a post reconstruction magnification equal to 2 was used in paediatric cases. In this way, 3D surface images of paediatric kidneys' were created by a final 4 fold magnification to obtain the best spatial detail. FBP, Hanning with crucial frequency 0.8 and ramp filter were used in renal reconstructions. Sheehy et al, 2009, compare two filtering methods (OSEM and FBP) of reconstructing renal SPECT studies and noted that both techniques yielded identical findings for 94 of the 98 kidneys evaluated.

Lyra et al 2001, use the planar and tomographic reconstructed images for the calculation of three indices that are the ratios of counts of upper to lower, upper to middle and lower to 
middle part by a tomographic reconstruction technique "by parts" and they offer a quantitative comparison of the planar and tomographic images.

3D surface images have not been evaluated up to now in an angular display of surface images either as normal or pathological 3D images, where scars, renal size and renal pelvis imprint can be identified. Following figures (fig. 5.1.1, 5.1.2, 5.1.3, 5.1.4) are examples of normal and abnormal situations of the kidneys in which different qualitative signs are emerged.

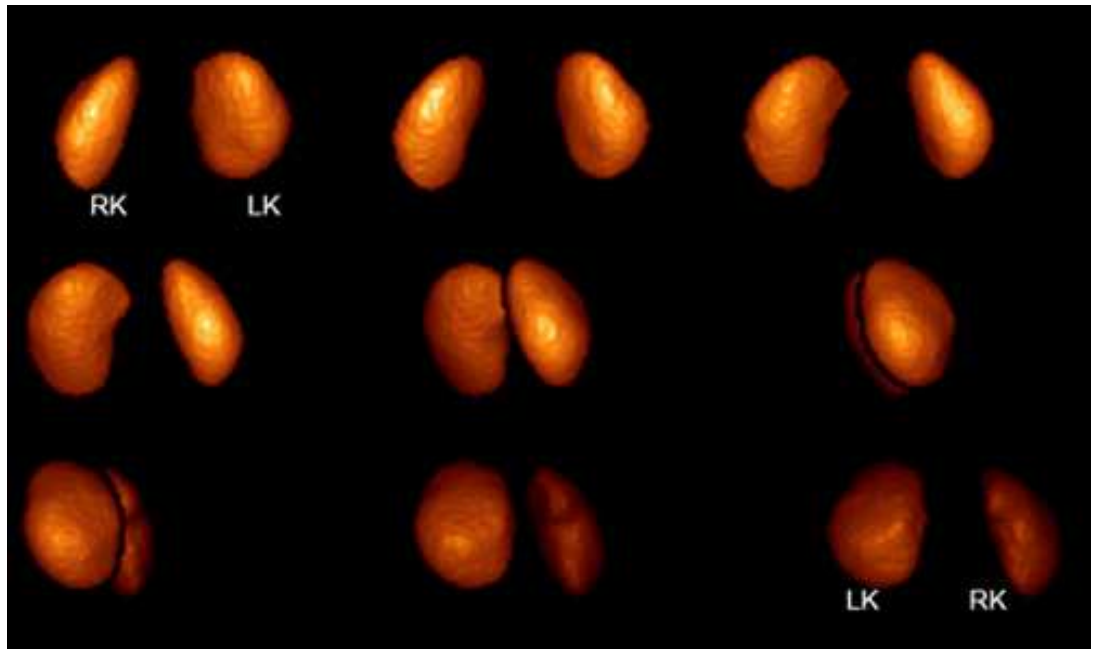

Fig. 5.1.1. 3D surface images of the normal kidneys of a child 1 year old, reconstructed for 9 angles around the body. Notice a tiny impress of right renal pelvis-Physiological sign-.

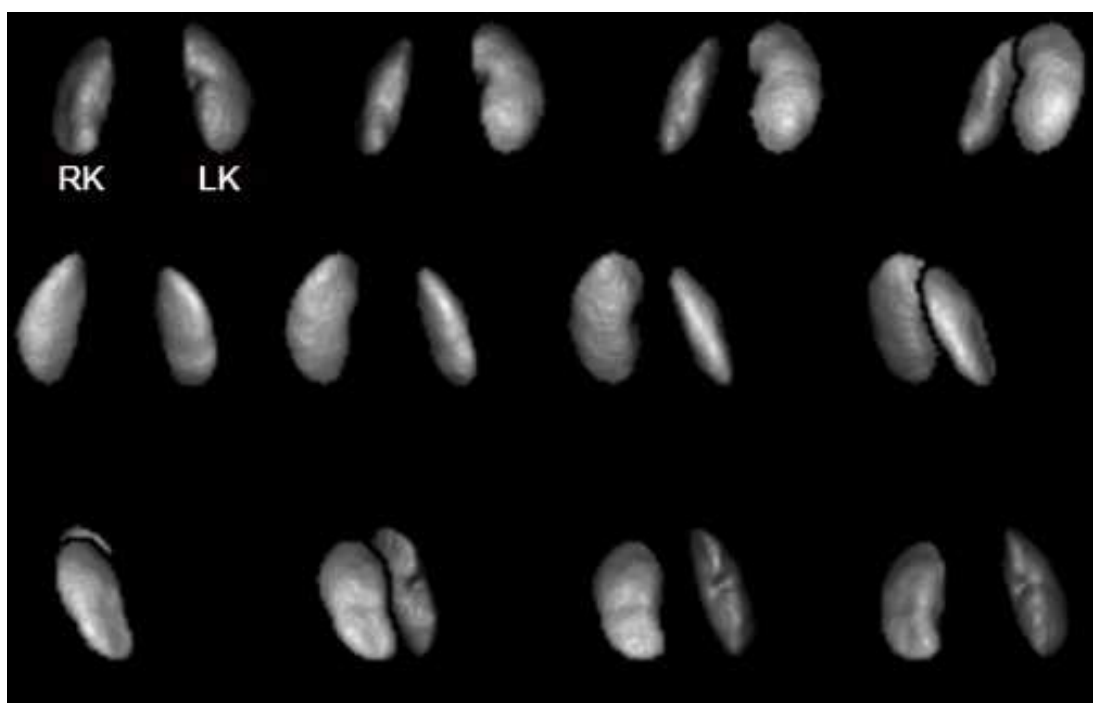

Fig. 5.1.2. 3D surface images at angular positions of a 6 months old renal study, in grey scale. Physiological impress of both renal pelvis. Reduced functioning parenchyma of the lower pole of right kidney 


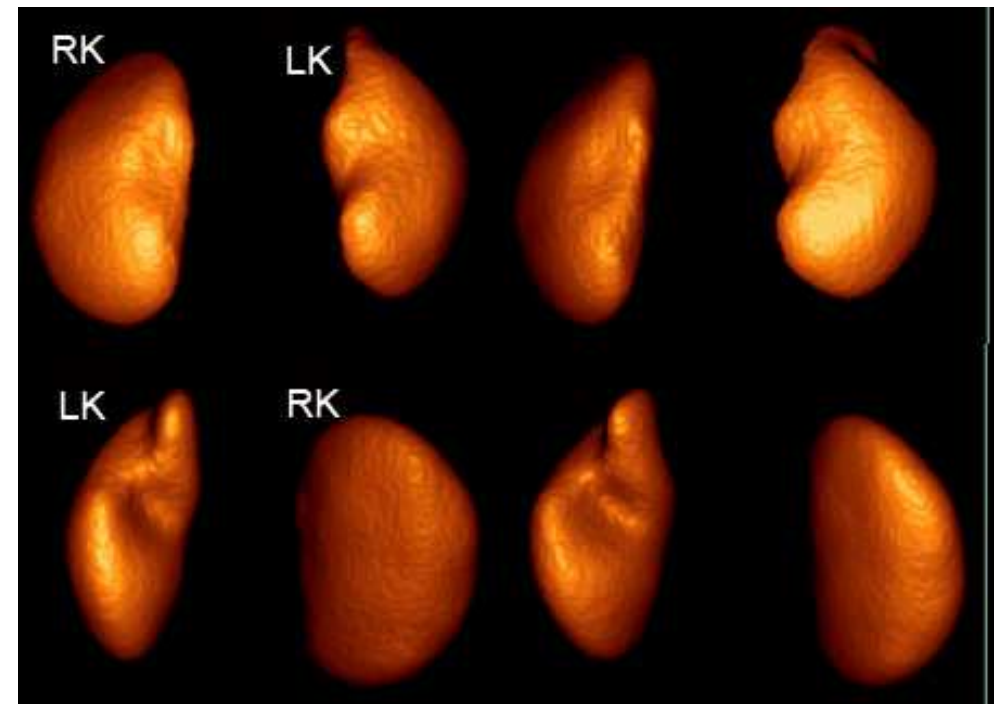

Fig. 5.1.3. 3D surface reconstructed angular images of kidneys; Deficiency of $18 \%$ of the upper pole of left kidney (scar) and central pyelocalyceal system -pyelonephritis-.

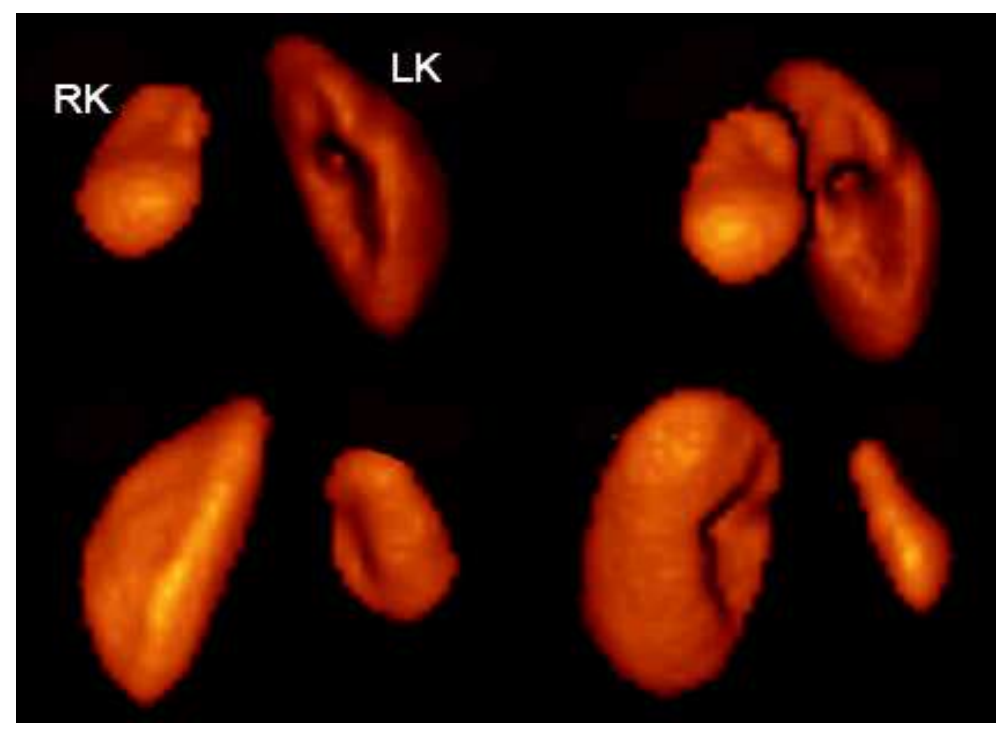

Fig. 5.1.4. 3D surface images at 4 angles; Compensatory hypertrophy of left kidney with important deficiency of the parenchyma close to pyelocalyceal system; Atrophic right kidney, with $22 \%$ of total functioning renal parenchyma.

3D display clearly offers additional valuable information. The procedure does not require any extra quantity of radiopharmaceutical to be injected to the patient, therefore the radiation burden is the same. The extra time required is in the order of 15 minutes, therefore not presenting any inconvenience for the patient. 


\subsection{Myocardium perfusion-3D surface images}

Heart scintigraphy provides information with respect to the detection of myocardial perfusion defects, the assessment of the pattern of defect reversibility and the overall detection of Coronary Artery Disease (CAD). There is a relationship between the location and the degree of the stenosis in coronary arteries and the observed perfusion on the myocardial scintigraphy, using data of 3D surface images of myocardium. This allows us to predict the impact evolution of these stenoses to justify a coronarography or to avoid it.

The visual interpretation of Tc99m tetrofosmin SPECT myocardial perfusion images can be challenging, due to the quantity of scan information generated by the large number of normal variants, attenuation artefacts and gender differences.( García-Panyella \& Susín 2002) Defects of the cardiac structure can be emerged from the $3 \mathrm{D}$ surface images of myocardium. Investigations on phantom studies (Matsunari et al, 2001) have been done and new method for segmentation of left ventricle (LV) for estimation of defects' size (Soneson et al, 2009) has been validated.

Our Cardiac patients had completed stress (Tc99m tetrofosmin at stress peak) and rest SPECT test by a GE Starcam 4000 tomographic gamma camera, use of $180^{\circ}$ arc rotation, step and shoot, $20 \mathrm{sec}$ per projection and 64×64 matrix size and magnification 2, for data acquisition. The data of the 2 sets (stress-rest) of slices were used to produce 3D surface images of myocardium for 16 angles around the body of the patient. We exploited the myocardial 3D scintigraphic data of the left ventricle, at stress and at rest, in order to recognize the cardiac volume and estimate perfusion defects as a percentage of $\mathrm{LV}$ myocardium mass. Co identification of myocardial perfusion images data was performed to eliminate normal morphological variances such as variances in orientation, size and shape, so that the remaining differences represent important functional differences. Dixon et al, 2004 suggest that when attenuation correction and detector resolution compensation are applied during reconstruction, patient variables do not influence the quantitative accuracy. A significant improvement in results was found with zoomed acquisitions.

$3 \mathrm{D}$ data reconstructed by FBP, obtained at stress and at rest scintigraphic studies, used to evaluate the left ventricle deformation in both stress - rest 3D surface image series. If a significant difference is obtained in rest and stress 3D data perfusion, the location and the impact of the pathology of left ventricle myocardium are recognized. The myocardial defects have been calculated as percentage of the myocardium at rest after the estimation of the total myocardium.

The following 5 next figures (fig 5.2.1, 5.2.2, 5.2.3, 5.2.4, 5.2.5) are referred to cases that myocardium diagnosis is rely on 3D surface shaded images; $3 \mathrm{D}$ data obtained at stress and at rest of the LV myocardium, respectively, are analysed and the deformation of both images is evaluated, qualitatively and quantitatively.

It is expected that further significant improvement in image quality will be attained, which, in turn, will increase the confidence of image interpretation. The development of algorithms for analysis of myocardial 3D images may allow better evaluation of small and non-trans mural myocardial defects. For the diagnosis and treatment of heart diseases the accurate visualisation of the spatial heart shape, 3D volume of the LV and the heart wall perfusion play a crucial role. Surface shading is a valuable tool for determining the presence, extent and location of CAD. 


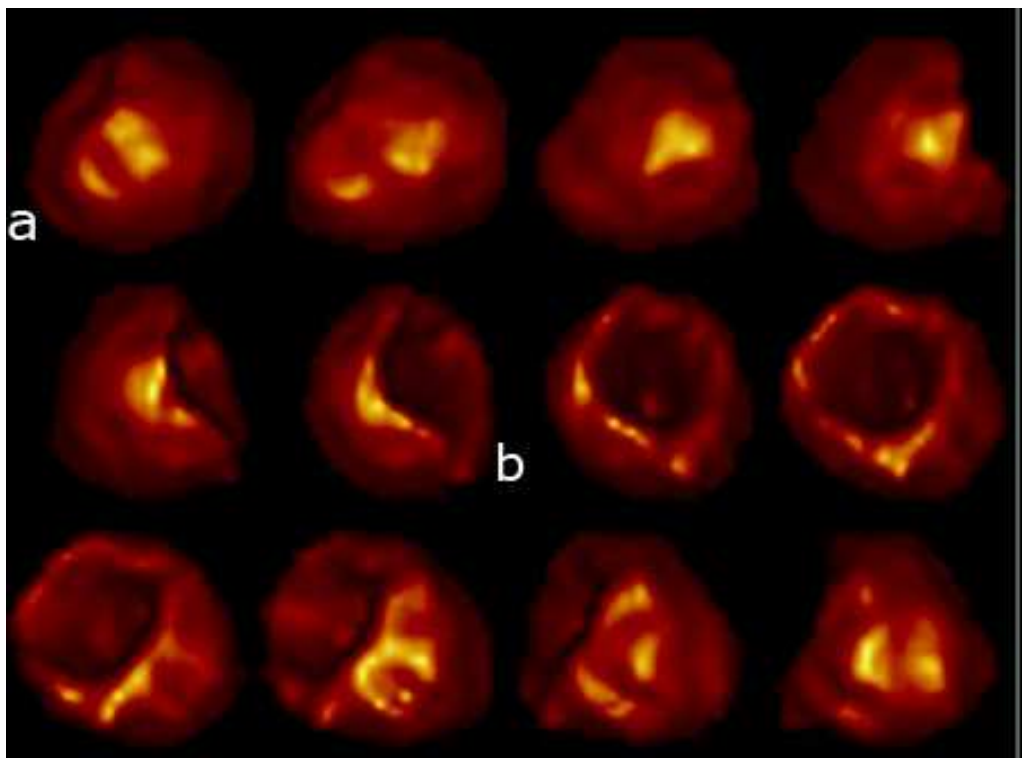

Fig. 5.2.1. 3D shaded surface display of a patient stress perfusion angular images. The study by Tc99m tetrofosmin shows normal myocardium perfusion. (a) for apex and (b) for base of myocardium. Transaxial slices were reconstructed and the created volume images show the apex at the left side. Through base we recognize the cavity of LV. No stress defect was observed and calculated as $0 \%$ of the total myocardium.

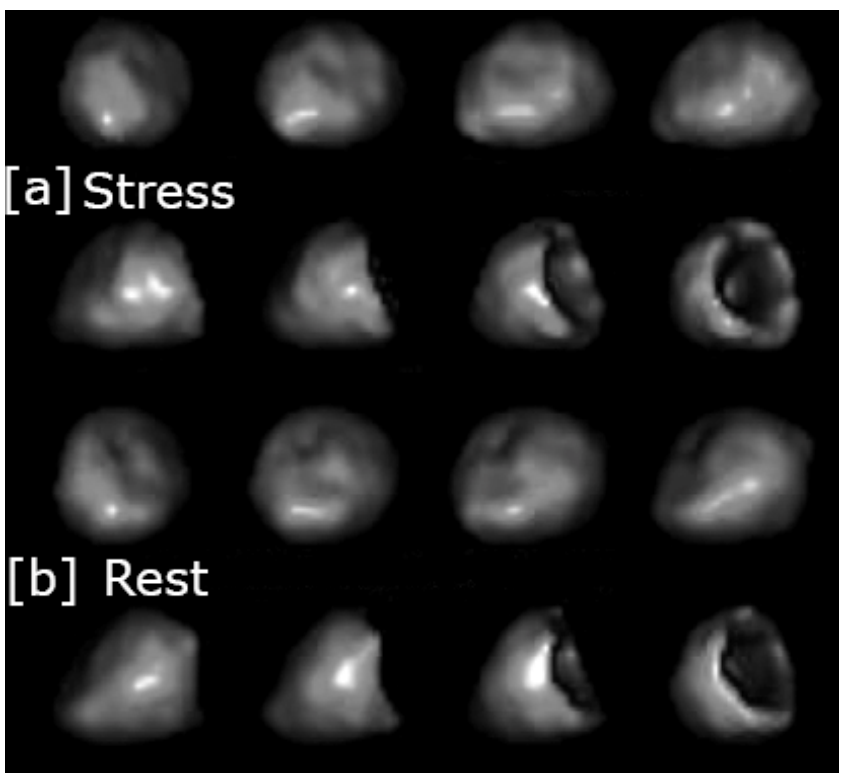

Fig. 5.2.2. Small defect at posterior- basal wall at stress (3\% of the myocardium). Partial improvement at rest ( $2 \%$ rest defect); Threshold value $50 \%$ of maximum. 


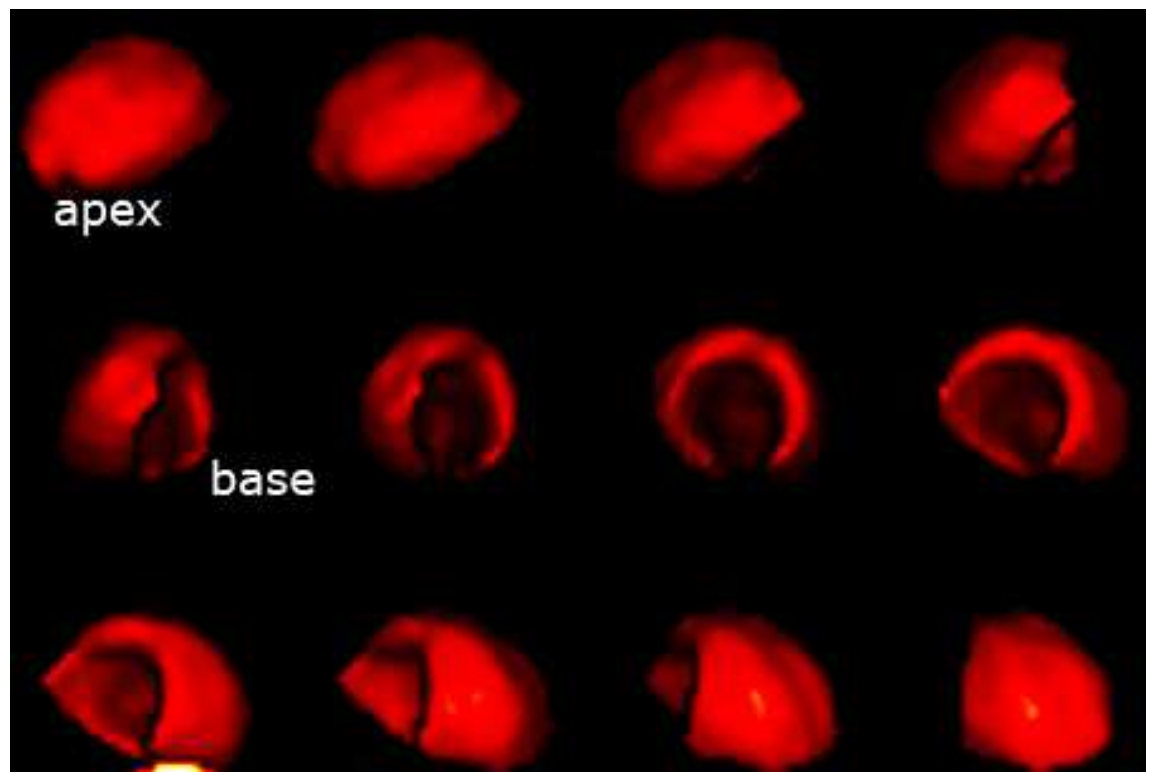

Fig. 5.2.3. 3D volume myocardium, at Rest. Permanent ischemic findings at inferior basal wall; Rest defect $8 \%$ of the total myocardium. Threshold: $50 \%$.

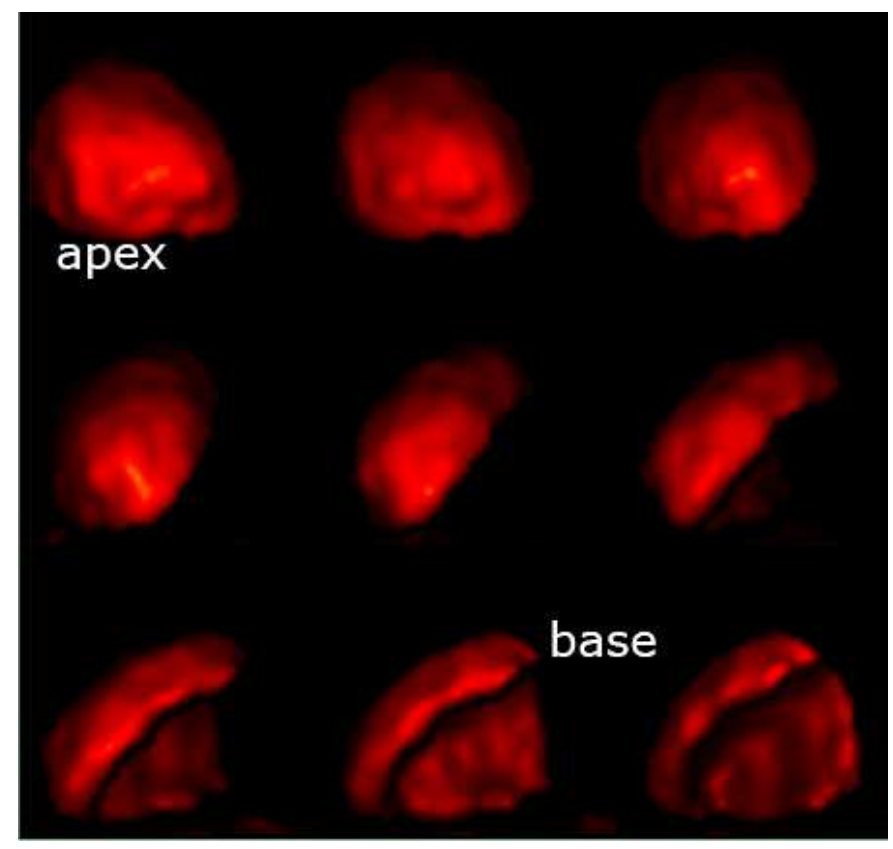

Fig. 5.2.4. 3D surface images of a male myocardium study at Rest. Permanent defect at inferior, inferior-posterior and lateral of LV. Rest defect: $28 \%$ of total myocardium volume. 


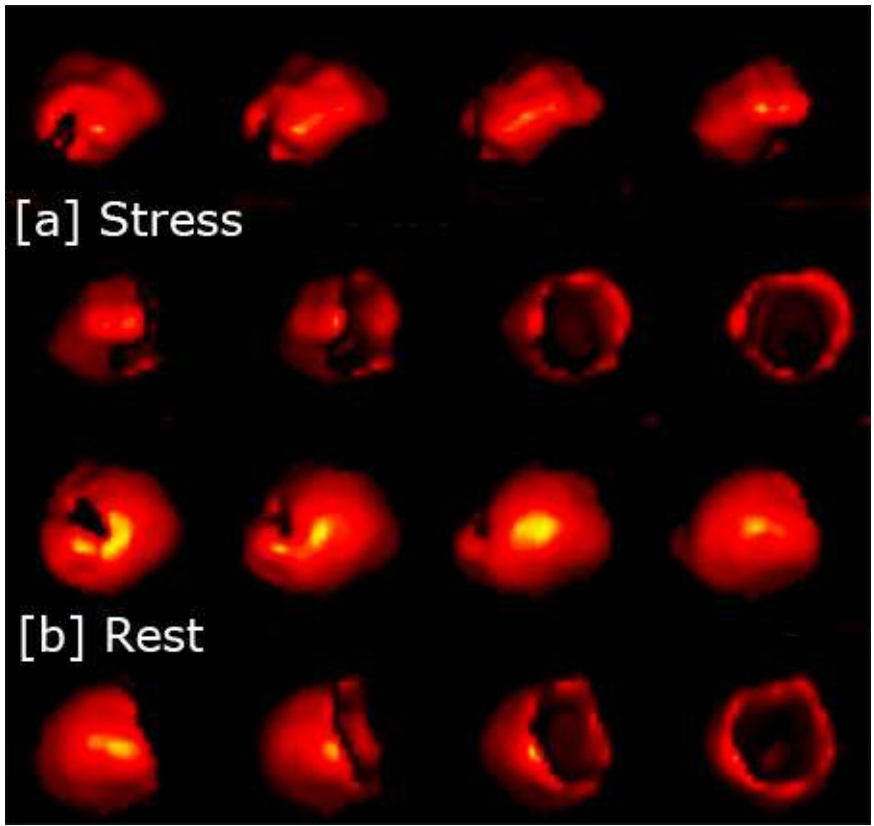

Fig. 5.2.5. Stress [a] and Rest [b] 3D surface angular images of female myocardium. Low extent permanent scar at apex wall and $4 \%$ defect at posterior - basic wall during stress. $\mathrm{O} \%$ defect at rest.

\subsection{Liver 3D surface images}

Patients on the suspicion of hepatocellular disease may complete a liver scan. They are injected intravenously with Tc-99m Phytate and images are acquired 10 minutes post injection. The acquisition involved four planar images and a tomographic study of 64 planar views over a $360^{\circ}$ arc. Each SPECT angle view is preset to end in $20 \mathrm{sec}$.

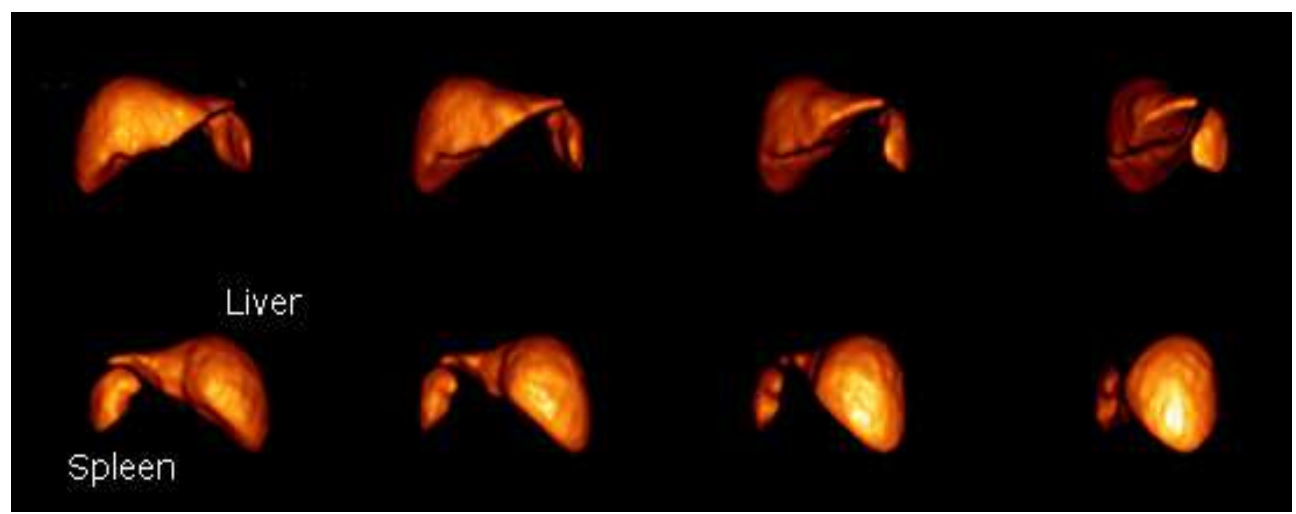

Fig. 5.3.1. 3D surface shading angular series images of liver and spleen of a 12 years old boy. Normal volume and smooth surface of both organs. 
The tomographic reconstruction is performed with the FBP, Hanning 0.8 and no magnification. An image threshold calculated according to equation 3 for each specific patient and a gradient-shading factor of $20 \%$ is applied on the 3D reconstructed angular images.

3D surface images of the liver could be presented together with the planar anterior image and series of coronal views to increase the diagnostic effectiveness of the method, as information of the surface texture and volume size of this large organ together with Spleen position and size express its patho-physiology. Shin et al, 2009 present surface models of detailed structures inside and outside the liver to promote medical simulation system of the abdomen.

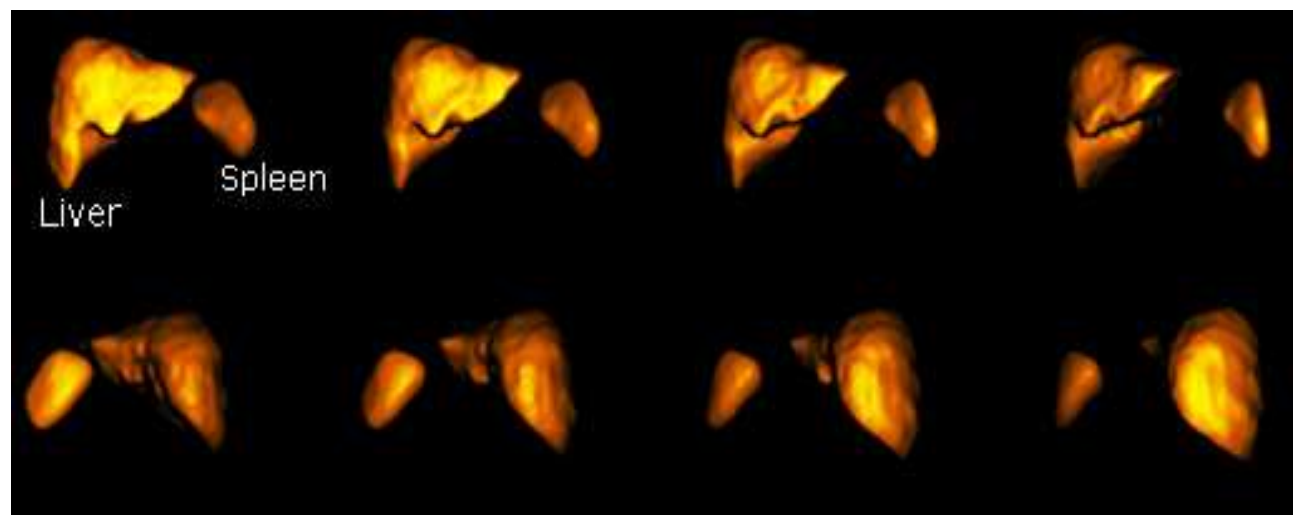

Fig. 5.3.2. 3D volume shading images of another normal variant of liver. Notice the impress of the gallbladder location in the middle of the liver, between right and left lobes (2-4 angular images).

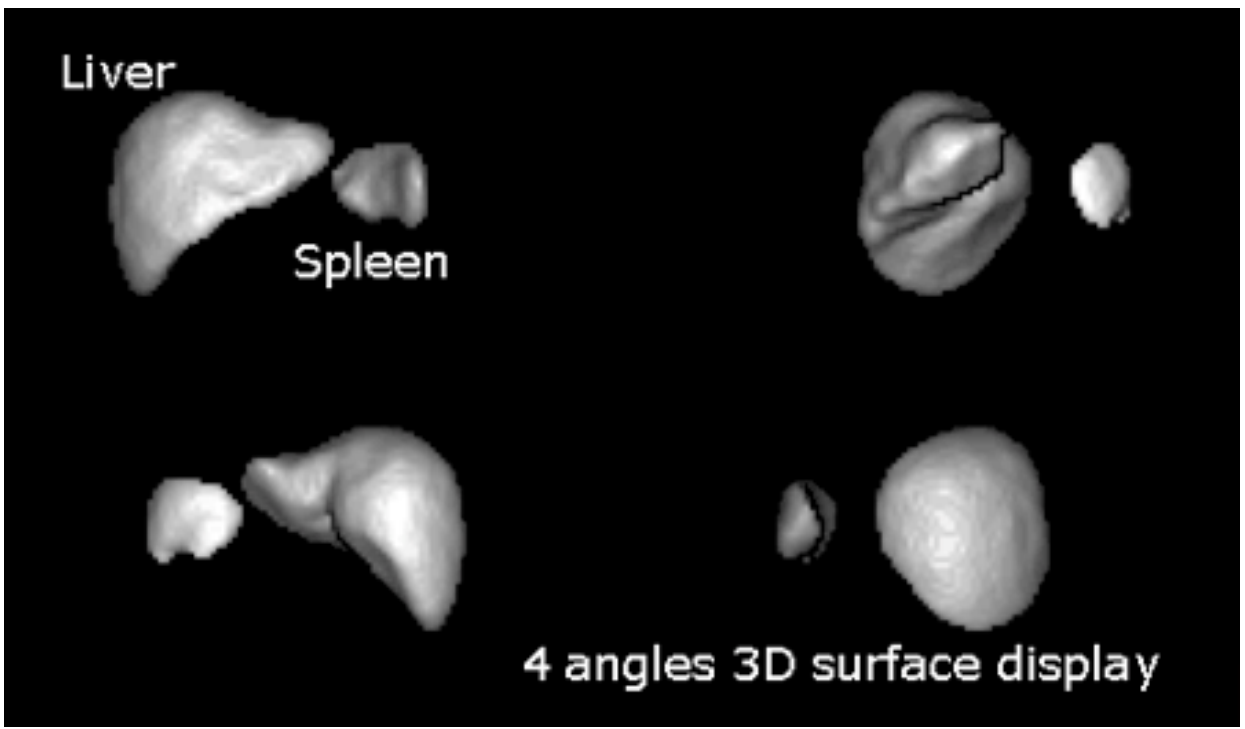

Fig. 5.3.3. 3D shading volume images of liver and spleen. Smooth surfaces of the organs. A part of the spleen is only presented (abnormal shape, image 1 and 4 ) due to rupture of the spleen at a recent car accident. 


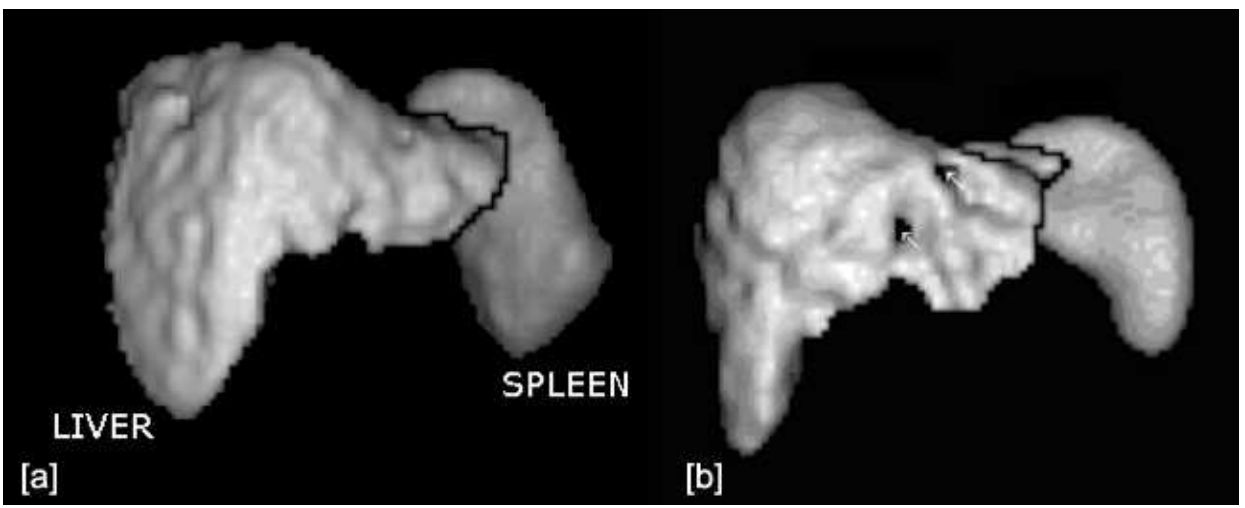

Fig. 5.3.4. The signs of the hepatocellular disease (non-homogeneous distribution in liver) are evident in the 3D volume images. Non smoothed surface of the liver (cirrhotic signs) and increased volume size of the spleen.

\subsection{Lung perfusion 3D surface images}

SPECT of perfusion lung study not only increases the diagnostic accuracy of the method but also permits the application of advanced image-processing techniques (Reinartz et al, 2006; Roach et al, 2008). In the evaluation of the lung status during pulmonary embolism and patient's follow up, accurate volume estimation is important. Lungs' 3D volume display, by

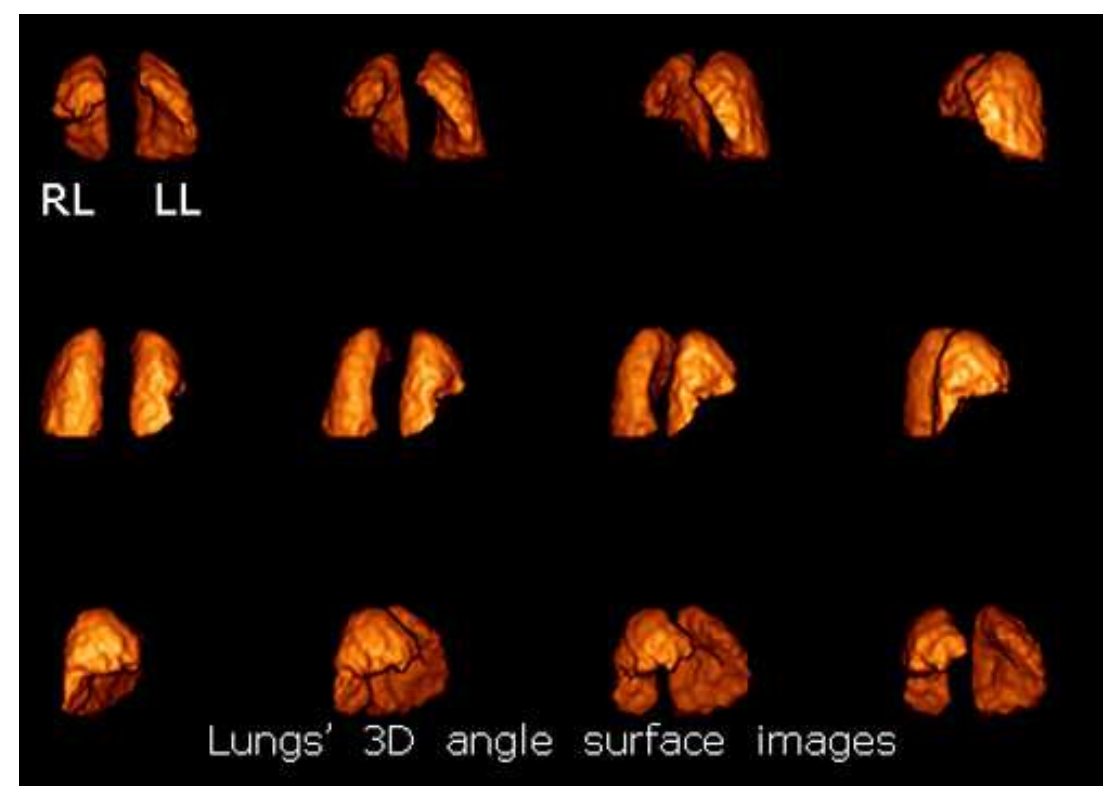

Fig. 5.4.1. Sequential 3D angular images of lungs' volume in a perfusion study. There is a large triangular defect in anterior basal and medial lobes of the right lung (RL) due to decreased blood perfusion caused by emboli (images 10,11). Notice the cavity at left lung that accommodate heart (image 12). 
reconstruction of SPECT images, can demonstrate the perfusion non-uniformity of the lungs' parenchyma.

Patterns of 3D perfusion scans with Tc-99m microspheres can provide an estimation of the extent of pulmonary embolism. Measurement of regional distribution of blood flow can help predict the consequences of lung resection or lung reduction surgery and cystic fibrosis, or radiation therapy. Four functional categories of lung pathology can be distinguished: the vascular occlusive state and the consolidative, obstructive, and restrictive states, resulting in scintigraphically detectable distortions of perfusion. Segmental or sub segmental hypo perfusion can be caused by obstruction of pulmonary vessels due to intra-or extra vascular pathology, including perfusion emboli.

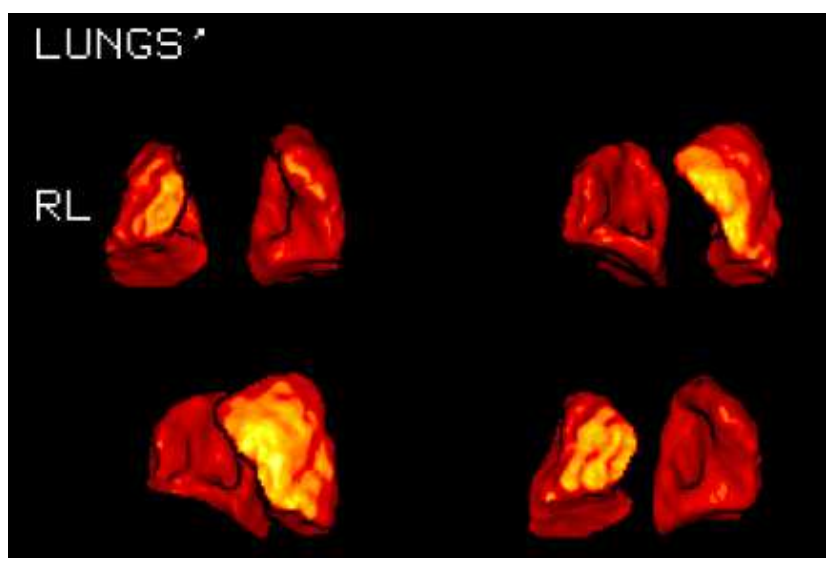

Fig. 5.4.2. 3D tomography emerges an anterior basal defect [11\%], of total volume, at right lung

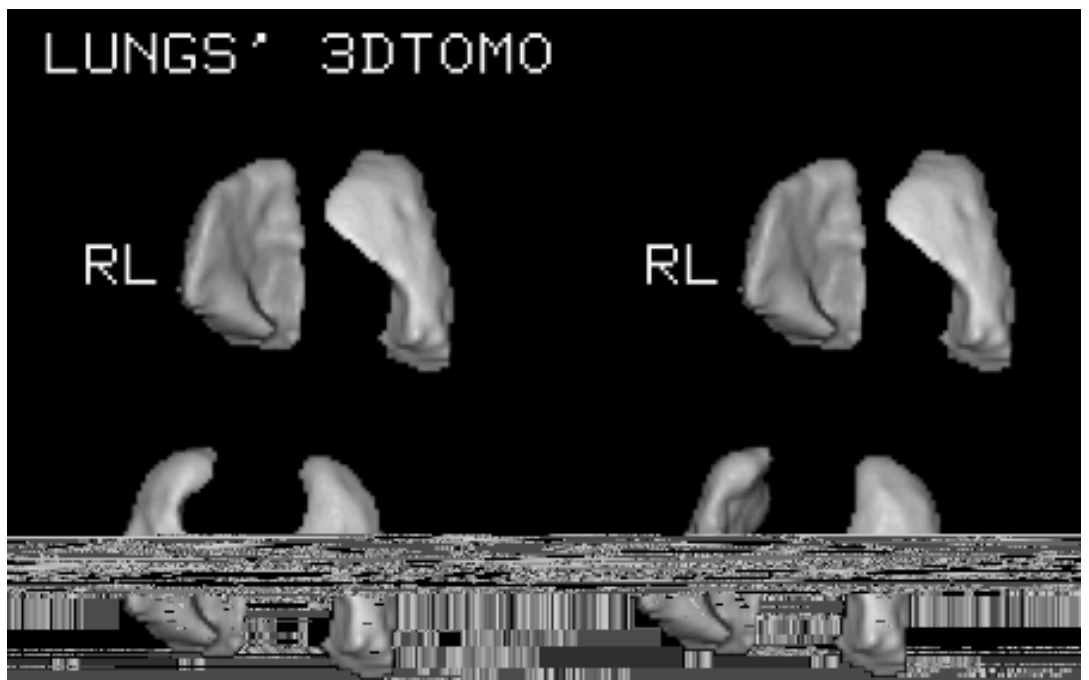

Fig. 5.4.3. Small triangular perfusion defect (5\%) at the superior lobe of left lung; Similarly, two small perfusion defects at superior and lateral -basal lobes of right lung ( $3 \%$ each). Multiple acute lungs embolisms 
A SPECT lung study can be used to estimate the lung perfusion improvement in details. Data reconstructed in transverse, coronal, sagittal slices as well as 3D surface images and series of follow up SPECT studies, after the pulmonary embolism event, must be used (Lyra et al, 2008b).

From lung perfusion SPECT data, volume of each reconstructed transverse slice can be estimated by counting the voxels inside each slice. The grey histogram edge detection program mark the lung lobe's edge and subtract background. Non-uniform attenuation compensation is performed by quantitative FBP.

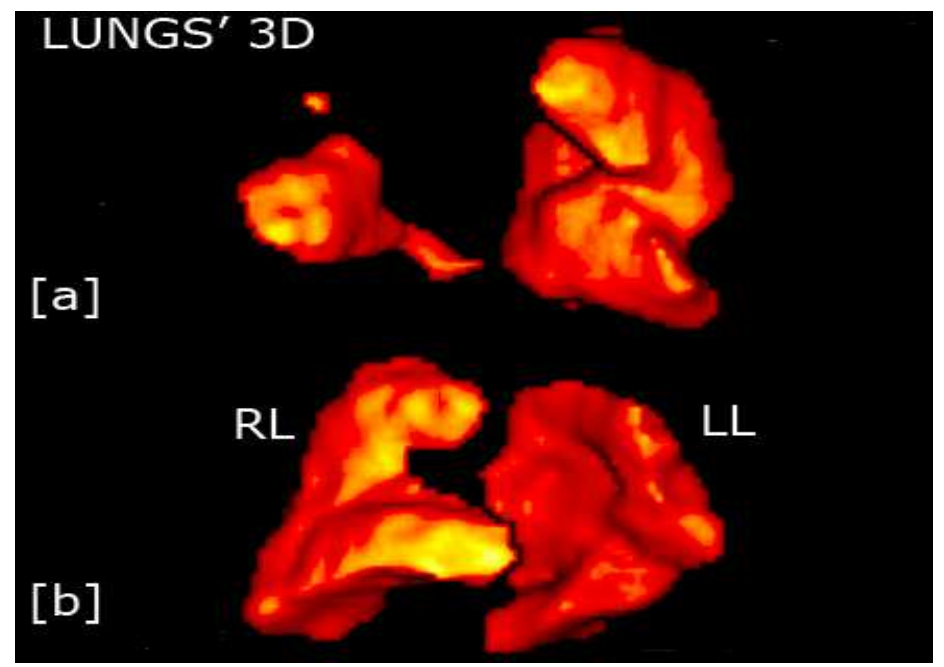

Fig. 5.4.4. Follow up 3D studies after the pulmonary embolism event, in order to estimate the lung perfusion improvement, quantitatively.

[a] Four hours post the event; Great right lobe (RL) embolism; Right Lobe volume: $23 \%$ of total Volume (both lungs)

[b] Eleven days post therapy; Right Lobe (RL) volume recovery : 49,6\% of total Volume

\subsection{Thyroid studies- 3D surface images}

Single photon emission tomography of the thyroid gland enables improved accuracy over planar imaging in the determination of the volume, regarding the thyroid configuration variants and the difficulty of definition of the organ's borders over the surrounding background.

The accuracy of the volume estimations depends on the correct delineation of the borders of the thyroid tissue. The thersholding procedure adopted is the grey histogram thresholding and is specific for each case, in a range that start even from $20 \%$ of its maximum. Difficulties arise due to various factors including fluctuations in the background and the gland's shape and function as well as an unavoidable smoothing of the thyroid boundary during reconstruction (Zaidi, 1996a).

Tomographic images at various levels are obtained with each slice 1 pixel thick. That is, the size of the elementary voxel is one pixel in the $x$ and $y$ axis and 1 pixel, too, in the $z$ direction. After correction for the contribution of background, the pixel values in a $2 \mathrm{D}$ transverse or coronal slice represent the radioisotope consentration within that slice. 


\section{OO} (0) 0 00

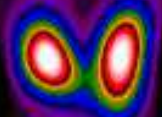

\section{Thyroid coronal slices}
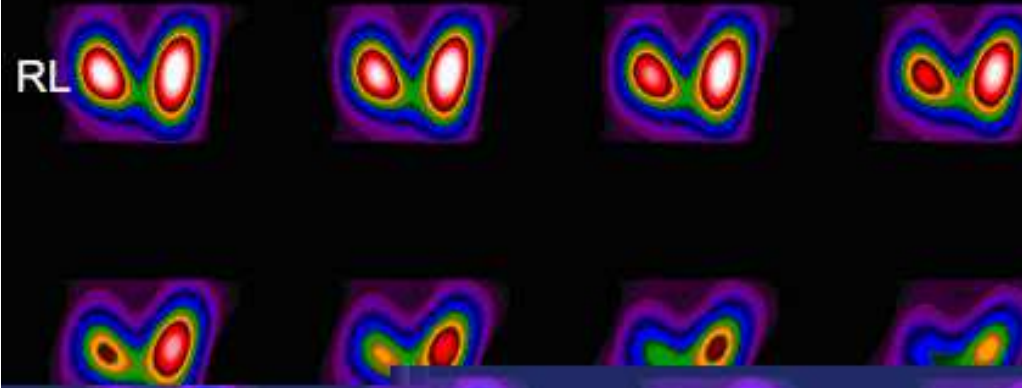

Fig. 5.5.1. A series of 16 coronal slices covering the thyroid gland thickness and showing the area of each slice.

Influence of attenuation compensation for a superficial small organ as the thyroid gland, seems not significant for volume measurements. The geometry - organ size, shape and location - varies between individuals. Volume surface displays of thyroid gland at angles show the real configuration of the gland's lobes.

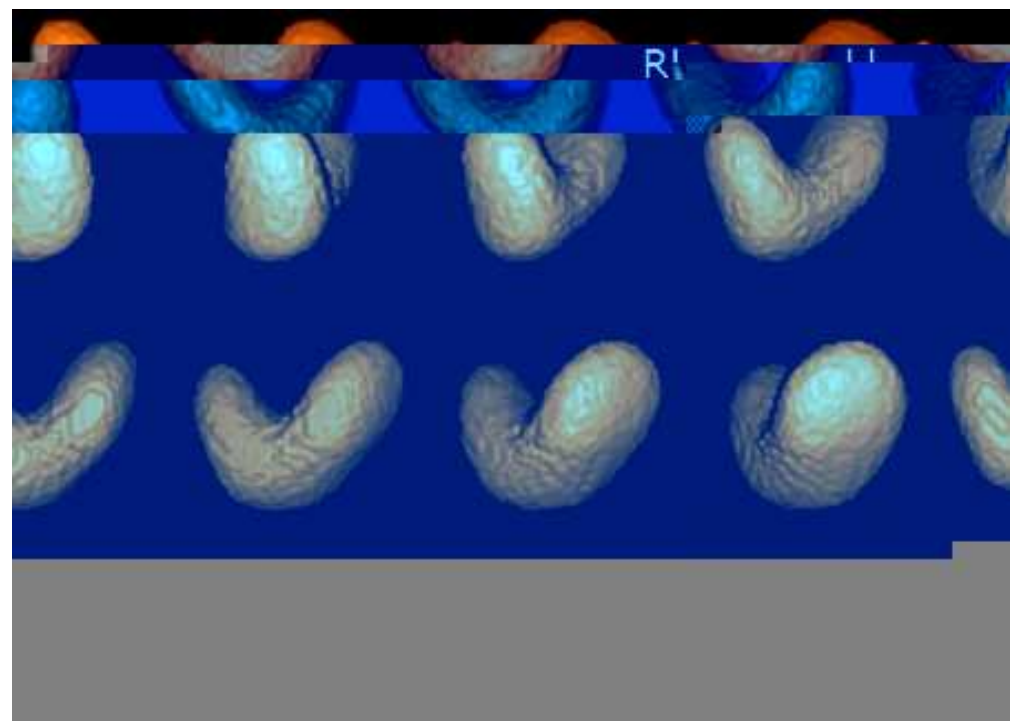

Fig. 5.5.2. 3D thyroid gland images at 16 various angles. Increased the right lobe size and high intensity at its upper pole showing a "hot" node. 


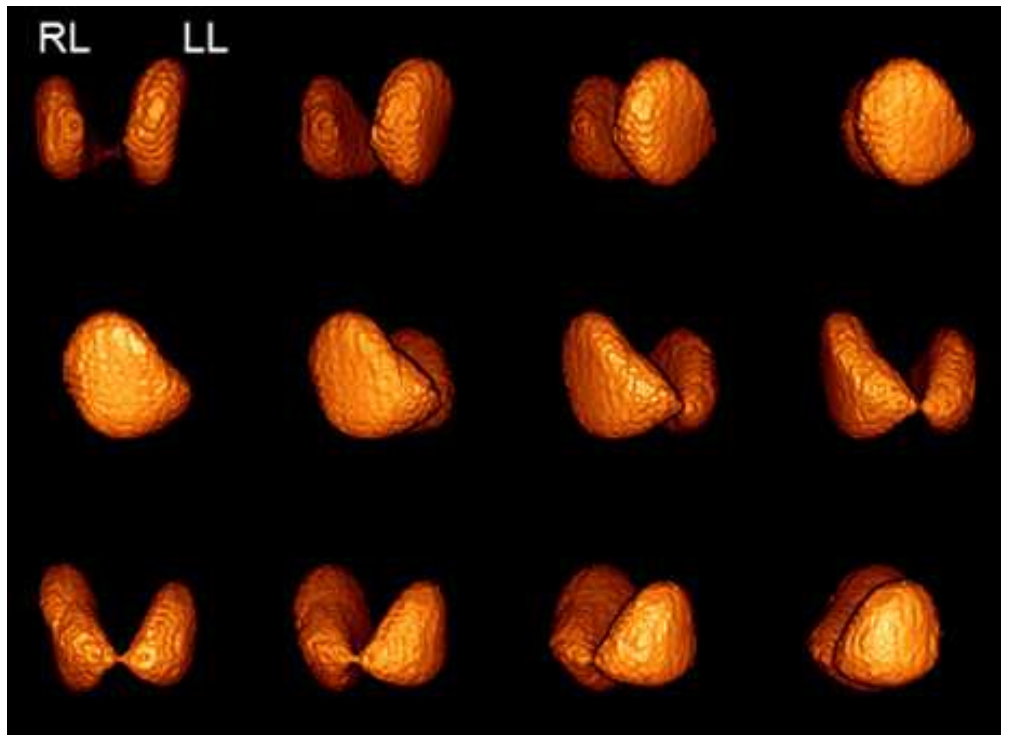

Fig. 5.5.3. Increased left lobe; butterfly shape with very thin isthus, impossible to be seen in planar scan.

A threshold to every single case is adapted as its value is dependent upon size of the imaged organ and the contrast. The thyroid lobes are enclosed with the help of regions of interest (ROI) tools for a first contouring its borders. Size of the organ, however, influences the correct threshold which correspond to a level slightly greater than the maximum fluctuation in the image background. After subtracting this level from the image, the boundary of the thyroid is delineated by the volume elements (voxels) that contain non zero counts; that is the border pixels contain counts equal to the threshold.

Gradient shading was used to surface images, by a gradient factor up to $5 \%$ low. Determination of thyroid volume leads to the calculation of the thyroid mass and the accurate activity to be administered for patient specific therapeutic purposes (Pant et al, 2003).

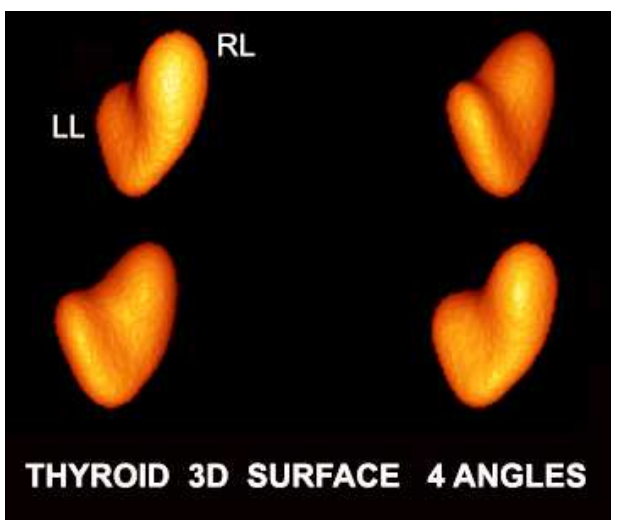

Fig. 5.5.4. Smoothed surface volume displays. Increased size of right lobe. The two lobes come close by a very thick isthus. 


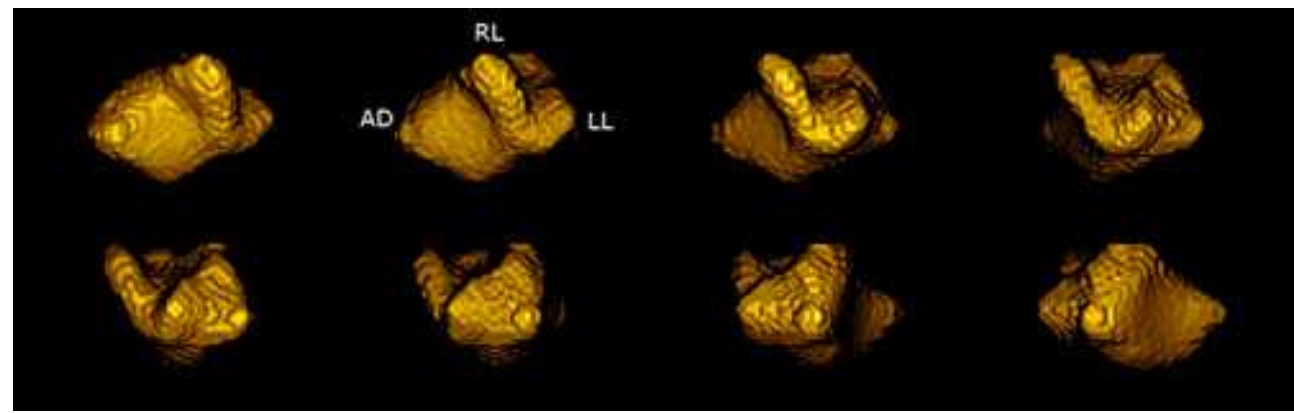

Fig. 5.5.5. The large toxic adenoma is appeared back to the right lobe. The two lobes could not be formed at all in the planar scan but are emerged in this 3D - low threshold-image.

\section{Conclusion}

By gathering sufficient volume data, analysis of 3D images gives valuable information to give the volume, shape and texture abnormalities of an organ (nodules in Thyroid, homogeneity of the liver surface) or defect regions' (emboli in Lungs, low perfusion in myocardium). Shading according to the gradient of the surface, results in 3D texture surface display, useful in pathologies (e.g. cirrhotic Liver).

Data sets from the above mentioned organs could also be analyzed by image processing software to take new valuable parameters. These images can be transferred in an uncompressed bitmap format and processed by Interactive Data Language (IDL) tools .The volume visualization of nuclear medicine data takes advantage of $3 \mathrm{D}$ texture analysis. So, in these $3 \mathrm{D}$ reconstructions, volumetric estimations and geometrical data measurements can be extracted; and undoubtedly they are useful in the diagnosis of organ morphological and functional abnormalities.

It is showed that 3D surface shaded images in angles, around the organ, are sensitive with the possibility to extract quantitative information for all organs that are studied. 3D imaging offers direct visualization and measurements of complex structures of the internal organs of the human body, which cannot be satisfactorily evaluated using 2D imaging.

As reconstruction methods improve in accuracy and in ability to manipulate large matrices, new threshold techniques and corrections will be used, so that perfect absolute quantitative accuracy will be brought closer.

\section{References}

Boudraa, A.O. and Zaidi, H. (2006) Image segmentation techniques in nuclear medicine imaging, In: Quantitative Analysis in Nuclear Medicine Imaging, Zaidi, H., (Ed.), (308357), Springer, ISBN: 978-0-387-23854-8, Heidelberg, Germany

Cooke C.D.; Faber, T.L.; Areeda, J.S. and Garcia, E.V. (2001) Advanced computer methods in cardiac SPECT, Cardiac SPECT imaging, De Puey, E.G., Garcia, E.V., Berman S.D. (ed), 65-80, Lippincott Williams \& Wilkins, ISBN 0-7817-2007-9,Philadelphia 
Csetverikov, D. (2007), http://visual.ipan.sztaki.hu/ELTEfoliak/1-in-page/lec07_ threshold_prn.pdf, Eötvös Loránd University,Department of Informatics, Budapest, Hungary

Daou, D.; Pointurier, I.; Coaguila, C.; Vilain, D.; Benada, A.W.; Lebtahi, R.; Fourme, T.; Slama, M.; Le Guludec, D. (2003) Performance of OSEM and depth-dependent resolution recovery algorithms for the evaluation of global left ventricular function in $201 \mathrm{~T} 1$ gated myocardial perfusion SPECT. J Nucl Med, Vol. 44, No. 2, 155-162, ISSN 0161-5505

De Sadeleer, C.; Bossuyt, A.; Goes E. and Piepsz A. (1996).Technetium-99m-DMSA SPECT in Normal Volunteers, J Nuc. Med, 1996, Vol.37, pp.1346-1349

Dixon, K.L.; Fung, A.; Celler, A. (2004) The effect of patient acquisition and reconstruction variables on myocardial wall thickness as measured from myocardial perfusion SPECT studies. Nuclear Science Symposium Conference Record, Vol.7, (16-22 Oct.2004), 4141-4145, DOI 10.1109/NSSMIC.2004.1466804

Erdi, Y.E.; Wessels, B.W.; Loew, M.H. and. Erdi, A.K. (1995). Threshold Estimation in Single Photon Emission Computed Tomography and Planar Imaging for Clinical Radioimmunotherapy . Cancer Research (Supplement) 55, December 1, 1995, 5823s.$5826 \mathrm{~s}$

Garcia, E.V.; Cooke C.D.; Areeda, J.S. (2001).Quantitative analysis of SPECT, Cardiac SPECT imaging, De Puey, E.G., Garcia, E.V., Berman S.D. (ed), 41-64, Lippincott Williams \& Wilkins, ISBN 0-7817-2007-9,Philadelphia

García-Panyella O.; Susín A. (2002). Left Ventricle Volume Estimation from 3D SPECT Reconstruction, IEEE Computers in Cardiology, Murray, A., (Ed.), vol. 29, 621-624

Gourion, D. and Noll, D. (2002) The inverse problem of emission tomography, Inverse Problems, Vol.18 (2002), pp.1435-1460

Groshar, D.; Moskovitz, B.; Issaq, E. and Nativ, O. (1997). Quantitative SPECT of DMSA uptake by the kidneys: Assessment of reproducibility. Kidney International, Vol. 52 (1997), pp. $817-820$

Hansen, C. L. (2002a). Digital image processing for clinicians, part I: Basics of image format, Journal of Nuclear Cardiology, Vol. 9, No. 3, 343-349

Hansen, C.L. (2002b). Digital image processing for clinicians, part II: Filtering, Journal of Nuclear Cardiology, Vol. 9, No. 4, 429-437

Kao, C.M. and Pan, X. (2000). Non-iterative methods incorporating priori source distribution and data information for suppression of image noise and artefacts in 3D SPECT, Phys. Med. Biol. 45 , pp.801-819

Lyra, M.; Skouroliakou, K.; Georgosopoulos, C.; Stefanides, C. and Jordanou, J. (2001) Single Photon Emission Computed Tomography and 3 Dimensional Quantitative Evaluation in Renal Scintigraphy, (2001) Lecture Notes in Computer Science, Medical Image Computing and Computer-Assisted Intervention - MICCAI 2001, LNCS 2208, W. Niessen and M. Viergever (Eds.):, (January 2001), 1222-1223, Springer Berlin Heidelberg, ISBN 978-3-540-42697-4, ISSN 0302-9743 1611-3349, DOI 10.1007/3-540-45468-3_171 
Lyra, M.; Striligas J.; Gavrilelli M.; Chatzigiannis, C.; Skouroliakou C. (2008a). Thyroid Volume determination by single photon tomography and 3D processing for activity dose estimation, IEEE International Workshop on Imaging Systems and Techniques - IST 2008, Chania, Greece, September 10-12, 2008

Lyra, M.; Gavrilelli M.; Kokona, G.; Skouroliakou, C. (2008b) Lungs SPECT image processing for volume and perfusion index estimation. BioInformatics and BioEngineering, BIBE 2008. 8th IEEE International Conference on, Athens, Oct. 2008

Matsunari, I.; Yoneyama, T.; Kanayama, S.; Matsudaira, M.; Nakajima, K.; Taki, J.; Nekolla, S.G.; Tonami, N.; and Hisada, K. (2001). Phantom Studies for Estimation of Defect Size on Cardiac 18F SPECT and PET: Implications for Myocardial Viability Assessment., J Nucl Med, Vol. 42 No. 10, 1579-1585

Mortelmans, L.; Nuyts, J.; Van Pamel, G.; Van den Maegdenbergh, V.; De Roo, M. and Suetens, P. (1986). A new thresholding method for volume determination by SPECT, Eur J Nucl Med Mol Imaging, Vol. 12, Numbers 5-6 , September 1986

Murli A.; D'Amore L.; Carracciuolo L.; Ceccarelli M.; Antonelli L. (2008). High performance edge-preserving regularization in 3D SPECT imaging, Parallel Computing , Vol. 34 , Issue 2, 115-132, ISSN 0167-8191, Elsevier Science Publishers B. V, February 2008

Noo, F. and Wagner, J.M. (2001). Image reconstruction in 2D SPECT with $180^{\circ}$ acquisition, Inverse Problems, 17, No 5, 1357-1371, PII:S0266-5611(1) 20858-2

Pan, X.; Kao, C.M.; Metz, C.; Kiselev, A.; Kiselev, E. . (2001). Image Reconstruction in 3D Short-Scan SPECT, http://cfi.lbl.gov/3D-2001/abstracts/07-02.pdf

Pan, X.; Sidky, E.Y.; Kao, C.M.; Zou, Y. and Metz, C.E. (2002). Short-scan SPECT imaging with non-uniform attenuation and 3D distance-dependent spatial resolution, Phys. Med. Biol., No.47, pp.2811-2833

Pant, G.S., Kumar, R., Gupta, A. K., Sharma, S.K., Pandey, A.K. (2003). Estimation of thyroid mass in Graves' disease by a scintigraphic method, Nuclear Medicine Communications, Vol.24, No.7, (July 2003), 743-748

Razifar, P., Sandström, M., Schneider, H., Långström, B., Maripuu, E., Bengtsson E., Bergström, M. (2005). Noise correlation in PET, CT, SPECT and PET/CT data evaluated using autocorrelation function: a phantom study on data, reconstructed using FBP and OSEM. Journal: Bio Medical Central (BMC): Medical Imaging 5(5), 2005 doi: 10.1186/1471-2342-5-5.

Reinartz, P., Kaiser, H.J., Wildberger, J.E., Gordji, C., Nowak, B. and Buell, U. (2006). SPECT imaging in the diagnosis of pulmonary embolism: Automated detection of match and mismatch defects by means of image-processing techniques, J Nucl Med, Vol. 47, No. 6, June 2006, 968-973

Roach, P. J., Bailey, D. L., Schembri, G. P. (2008), Reinventing ventilation/perfusion lung scanning with SPECT, Nucl Med Communications, Volume 29, (12), December 2008, 1023-1025, ISSN: 0143-3636, DOI: 10.1097/MNM.0b013e328315efa1

Rosenthal, M.S.; Cullom, R.J.; Hawkins, W.; Moore, S.C.; Tsui, B.M.W. and Yester, M. (1995) Quantitative SPECT Imaging: A Review and Recommendations by the Focus 
Committee of the Society of Nuclear Medicine Computer and Instrumentation Council, J Nuc Med; 36:1489-1513

Russ, J.C. (2006). The image processing handbook, CRC Press, Taylor \& Francis group inc., ISBN 0-8493-2516-1, $5^{\text {th }}$ edition, Boca Raton, Florida

Sainz, M.; Susin, A and Bagherzadeh N. (2003) MTMesh Image Based Mesh Reconstruction and Rendering IAESTED Conference in Visualization, Imaging and Image Processing (VIIP'03), Hamza, M.H., (Ed.), 785-790

Sheehy, N.; Tetrault, T.A.; Zurakowski, D.; Vija, A.H.; Fahey, F. H. and Treves, S.T. (2009) Pediatric 99mTc-DMSA SPECT performed by using Iterative Reconstruction with Isotropic Resolution Recovery: Improved Image Quality and Reduced Radiopharmaceutical Activity, Radiology, No.251, ( March 2009), 511-516

Shin, D.S.; Chung, M.S.; Lee, J.W.; Park, J.S.; Chung, J.; Lee, S.B. and Lee, S.H. (2009). Advanced Surface Reconstruction Technique to Build Detailed Surface Models of the Liver and Neighbouring Structures from the Visible Korean Human, Korean Med Sci. , June 2009 , Vol.24, No.3, pp.375-383

Son, H.K.; Yun, M.J.; Jeon, T.J.; Kim, D.O.; Jung, H.J.; Lee, J.D.; Yoo, H.S.; Kim, H.J. (2001) ROC analysis of ordered subset expectation maximization and filtered back projection technique for FDG-PET in lung cancer. Nuclear Science Symposium Conference Record, 2001 IEEE, Vol. 3, Issue, 2001, 1801 - 1805, DOI 10.1109/NSSMIC.2001.1008692

Soneson, H.; Ubachs, J.F.A.; Ugander, M.; Arheden, H.and Heiberg E. (2009). An Improved Method for Automatic Segmentation of the Left Ventricle in Myocardial Perfusion SPECT. J Nucl Med, Vol.50, No.2, (February 2009), 205-213

Temiz, Y.; Tarcan, T.; Onol, F.F.; Alpay, H. \& Simsek, F.,(2006). The efficacy of Tc99m dimercaptosuccinic acid (Tc-DMSA) scintigraphy and ultrasonography in detecting renal scars in children with primary vesicoureteral reflux (VUR), International Urology and Nephrology (2006) Vol.38, pp.149-152

Tsui, B.M.W.; Zhao, X.; Frey E.C. and McCartney W.H. (1994). Quantitative single-photon emission computed tomography: Basics and clinical considerations. Seminars in Nuclear Medicine, Vol. XXIV, No 1, (January 1994), 38-65

Yang, J.Y.; Yang, J.A.; Seo, J.W.; Lee, S.J. (2001). The Diagnostic Value of 99mTc DMSA Renal Scan SPECT Images in Addition to Planar Image in Children with Urinary Tract Infection, J Korean Soc Pediatr Nephrol., Vol.5,No.1, (April 2001),.22-29

Yan, Y., and Zeng, L.G. (2008). Scatter and Blurring Compensation in Inhomogeneous Media Using a Postprocessing Method, International Journal of Biomedical Imaging, Vol. 2008,(December 2008) Article ID 806705, Hindawi Publ. Corp., doi:10.1155/2008/806705

Zaidi, H., (1996a) Comparative Methods for Quantifying Thyroid Volume Using Planar Imaging and SPECT, J Nuc. Med, Vol. 37, pp.1421-1426

Zaidi, H. (1996b). Organ volume estimation using SPECT. IEEE Transactions Nuclear Science, Vol.43, Issue 3, June 1996, 2174-2182

Zaidi, H. and Hasegawa, B. (2003) Determination of the Attenuation Map in Emission Tomography, J Nucl Med, Vol. 44 No. 2, 291-315 
Zingerman, Y.; Golan, H. and Moalem, A. (2009). Spatial linear recovery coefficients for quantitive evaluations in SPECT, Nuclear Instruments and Methods in Physics Research Section A: Accelerators, Spectrometers, Detectors and Associated Equipment, Vol. 602, Issue 2, 21 April 2009, 607-613 


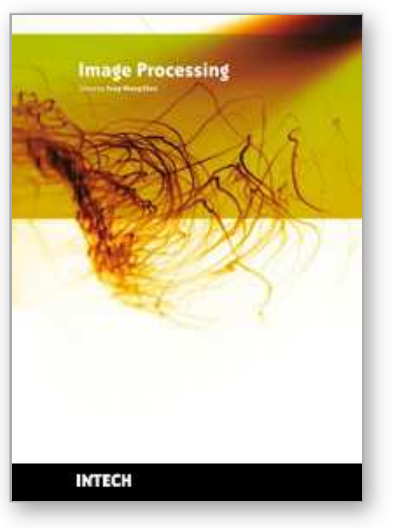

\author{
Image Processing \\ Edited by Yung-Sheng Chen
}

ISBN 978-953-307-026-1

Hard cover, 516 pages

Publisher InTech

Published online 01, December, 2009

Published in print edition December, 2009

There are six sections in this book. The first section presents basic image processing techniques, such as image acquisition, storage, retrieval, transformation, filtering, and parallel computing. Then, some applications, such as road sign recognition, air quality monitoring, remote sensed image analysis, and diagnosis of industrial parts are considered. Subsequently, the application of image processing for the special eye examination and a newly three-dimensional digital camera are introduced. On the other hand, the section of medical imaging will show the applications of nuclear imaging, ultrasound imaging, and biology. The section of neural fuzzy presents the topics of image recognition, self-learning, image restoration, as well as evolutionary. The final section will show how to implement the hardware design based on the SoC or FPGA to accelerate image processing.

\title{
How to reference
}

In order to correctly reference this scholarly work, feel free to copy and paste the following:

Maria Lyra (2009). Single Photon Emission Tomography (SPECT) and 3D Images Evaluation in Nuclear Medicine, Image Processing, Yung-Sheng Chen (Ed.), ISBN: 978-953-307-026-1, InTech, Available from: $\mathrm{http}: / / w w w . i n t e c h o p e n . c o m / b o o k s / i m a g e-p r o c e s s i n g / s i n g l e-p h o t o n-e m i s s i o n-t o m o g r a p h y-s p e c t-a n d-3 d-$ images-evaluation-in-nuclear-medicine

\section{INTECH}

open science | open minds

\author{
InTech Europe \\ University Campus STeP Ri \\ Slavka Krautzeka 83/A \\ 51000 Rijeka, Croatia \\ Phone: +385 (51) 770447 \\ Fax: +385 (51) 686166 \\ www.intechopen.com
}

\author{
InTech China \\ Unit 405, Office Block, Hotel Equatorial Shanghai \\ No.65, Yan An Road (West), Shanghai, 200040, China \\ 中国上海市延安西路65号上海国际贵都大饭店办公楼 405 单元 \\ Phone: +86-21-62489820 \\ Fax: +86-21-62489821
}


(C) 2009 The Author(s). Licensee IntechOpen. This chapter is distributed under the terms of the Creative Commons Attribution-NonCommercialShareAlike-3.0 License, which permits use, distribution and reproduction for non-commercial purposes, provided the original is properly cited and derivative works building on this content are distributed under the same license. 